\title{
TOPOLOGICAL SPACES OF PERSISTENCE MODULES AND THEIR PROPERTIES
}

\author{
PETER BUBENIK AND TANE VERGILI
}

\begin{abstract}
Persistence modules are a central algebraic object arising in topological data analysis. The notion of interleaving provides a natural way to measure distances between persistence modules. We consider various classes of persistence modules, including many of those that have been previously studied, and describe the relationships between them. In the cases where these classes are sets, interleaving distance induces a topology. We undertake a systematic study the resulting topological spaces and their basic topological properties.
\end{abstract}

\section{INTRODUCTION}

A standard tool in topological data analysis is persistent homology [30, 13, 29, 20, 41]. It is often applied as follows. One starts with some data, constructs an increasing family of complexes or spaces, and applies homology with coefficients in some fixed field to obtain a persistence module. Next, one computes a summary (e.g. barcode [22], persistence diagram [21], or persistence landscape [7, 11]) which determines this persistence module up to isomorphism. In practice, one computes these summaries directly from the increasing family of complexes or spaces. Nevertheless, the persistence module is the central algebraic object in this pipeline, and has been a focus of research.

A key discovery in the study of persistence modules is the notion of interleaving [15] which provides a way of measuring the distance between persistence modules. For many persistence modules, this distance equals the bottleneck distance [21] between the corresponding persistence diagrams [31, 12]. Interleavings and the resulting interleaving distance have been extensively studied both for the persistence modules considered here [31, 12, 1, 2, 18, 8, 15], for Reeb graphs [26, 35], for zig-zag persistence modules [6], for multiparameter persistence modules [31], and for more general persistence modules [9, 10, 27, 3, 33, 32].

For sets of persistence modules, the interleaving distance induces a topology. The main goal of the research reported here is to study the basic topological properties of the resulting topological spaces.

Unfortunately, this research program runs into an immediate difficulty: the collection of persistence modules is not a set, but a proper class. While it is possible the consider this class with the interleaving distance [9, 10, 8], here we want to work with actual topological spaces.

So to start, we consider various classes of persistence modules. These include classes that have been previously considered in theoretical work, such as pointwise finite-dimensional persistence modules [23], q-tame persistence modules [19], interval-decomposable persistence modules, ephemeral persistence modules [17], and constructible persistence modules [37, 25], as well as classes of persistence modules that arise in applications, such as those decomposable into finitely many interval modules, where each interval lies in some fixed bounded closed interval. 
We determine various relationships between these classes, such as inclusion (Figure 1). We also identify pairs of classes where for each element of one, there is an element of the other that has interleaving distance 0 from the first (Section 3.3). We define and calculate an asymmetric distance we call enveloping distance that measures how far one needs to expand a given class to include another (Section 3.4). These two results are summarized in Figure 2.

Next, we determine which of these classes are sets and which are proper classes. We show that the classes of interval-decomposable persistence modules and q-tame persistence modules are not sets (Corollary 3.27), though the classes of pointwise finite-dimensional persistence modules and persistence modules decomposable into countable-many interval modules are sets (Propositions 3.22 and 3.25). We introduce a set of persistence modules containing these two sets that consists of persistence modules decomposable into a set of interval modules with cardinality of the continuum (Definition 3.28 and Proposition 3.29 ).

For the remainder, we restrict ourselves to the identified sets of persistence modules and the topologies induced by the interleaving distance (Figure 3). We identify which of the inclusions in Figure 3 are inclusions of open sets (Proposition 4.1).

We show that these topological spaces are large and poorly behaved in the following ways. They do not have the $T_{0}$ or Kolmogorov property (Corollary 4.6), they are not locally compact (Corollary 4.9), and their topological dimension is infinite (Corollary 4.38). In fact, we prove the following.

Theorem 1.1 (Cube Theorem (Theorem 4.37)). Let $N \geq 1$. There exists an $\varepsilon>0$ such that there is an isometric embedding of the cube $[0, \varepsilon]^{N}$ with the $L^{\infty}$ distance into each of our topological spaces of persistence modules.

On the other hand, our topological spaces of persistence modules do have the following nice properties. They are paracompact (Lemma 4.10), first countable (Lemma 4.21), and are compactly generated (Lemma 4.22).

We determine which of these topological spaces are separable (Theorems 4.18 and 4.20), as well as second countable and Lindelöf (Lemma 4.23). We show that the space of pointwise finite-dimensional persistence modules is not complete (Theorem 4.24), but that the space of persistence modules that are both q-tame and that decompose into countably-many intervals is complete (Theorem 4.25). We prove a Baire category theorem for complete extended pseudometric spaces (Theorem 4.35) that implies that this space is also a Baire space (Corollary 4.36).

We also identify the path components of the zero module in our topological spaces (Propositions 4.14 and 4.16), and show that they are contractible (Proposition 4.17).

Along the way, we observe the following mild strengthening of the structure theorem for persistent homology [17], which may be of independent interest.

Theorem 1.2 (Structure Theorem (Theorem 3.8)). The radical of a q-tame persistence module is a countable direct sum of interval modules.

Persistence modules and persistence diagrams Topological data analysis tends to focus on persistence diagrams [21] rather than persistence modules. Readers more familiar with persistence diagrams may wonder why we work with persistence modules and what our results imply for persistence diagrams.

Let us present three responses. First, persistent homology produces persistence modules. In many but not all cases, these persistence modules may be represented by a persistence 
diagram. Mathematically, persistence modules are the fundamental object of study. Second, one of our main motivations was to develop a theory that could be extended to multiparameter persistence modules [14, 31] and generalized persistence modules [9, 27, 10]. In this more general setting there is no hope for an analog of the persistence diagram. Third, our results for persistence modules may be used to obtain results for persistence diagrams as corollaries.

To be more precise, consider persistence modules that are pointwise finite-dimensional (see Section 3.1) with the interleaving distance. This forms an extended pseudometric space that we label (pfd). If we take the quotient obtained by identifying persistence modules with zero interleaving distance, then we obtain an extended metric space that is isometric with a space of persistence diagrams with the bottleneck distance [21]. This is the celebrated isometry theorem [16, 31, 18, 1, 12. Call this extended metric space (pd).

Now (pd) inherits many of the properties of (pfd). Specifically, it is not totally bounded, any element of $(\mathrm{pd})$ does not have a compact neighborhood, it is not path connected, the path component of the empty persistence diagram consists of persistence diagrams without points with infinite persistence, and this path component is contractible. Furthermore, (pd) is not separable and is not complete. In addition, for each $N$ there is an $\varepsilon>0$ such that there is an isometric embedding of the $N$-cube with diameter $\varepsilon$ and the $L^{\infty}$ distance into $(\mathrm{pd})$. So the topological dimension of $(\mathrm{pd})$ is infinite.

For the data scientist For the reader primarily interested in topological data analysis, we would summarize our results by stating that the extended metric space of persistence diagrams with the bottleneck distance is "big". Say we fix $c<d$ and restrict ourselves to persistence diagrams with finitely many points $\left(a_{i}, b_{i}\right)$ each of which satisfies $c \leq a_{i}<b_{i} \leq d$. This is a metric space. However, every neighborhood of every persistence diagram in this metric space is not compact. Also, the topological dimension of this metric space is infinite.

In order to apply certain statistical and machine learning tools, one may be tempted to start with a compact set of persistence diagrams. In light of these results, this is a drastic step.

Extended pseudometric spaces The results presented here for extended pseudometric spaces are straight-forward extensions of the standard results for metric spaces (Lemmas4.10, 4.21, 4.22, and 4.23 and Theorem 4.35). However, in order to keep the material accessible to applied mathematicians without a background in point-set topology, we include the proofs.

Related work Mileyko, Mukherjee, and Harer [34] consider the set of persistence diagrams with countably many points in $\mathbb{R}^{2}$ together with the topology induced by the $p$-Wasserstein distance for $1 \leq p<\infty$. They show that the subspace consisting of persistence diagrams with finite distance to the empty persistence diagram is complete and separable. We show the corresponding space for the bottleneck distance $(p=\infty)$ is complete (Theorem 4.25) but not separable (Theorem 4.20). In a subsequent paper with Turner [40] they study geometric properties of the same set with a slightly different metric.

Blumberg, Gal, Mandell, and Pancia [4] show that the set of persistence diagrams with finitely many points with the bottleneck distance is separable and that its Cauchy completion is separable. This completion is the set of persistence diagrams with the property that for every $\varepsilon>0$ there are only finitely many points with persistence at least $\varepsilon$. 
The authors have been informed of related work that is in preparation. Perea, Munch, and Khasawneh [38] have characterized (pre)compact sets of persistence diagrams with the bottleneck distance. Their results imply that compact sets have empty interior. Cruz [24] has results on metric properties for generalized persistence diagrams with interleaving distance.

Organization of the paper In Section 2, we provide background on persistence modules, indecomposable modules, interleaving distance, and pseudometric spaces. In Section 3, we define the classes of persistence modules that we consider, study the relationships between them, and identify which of them are sets. In Section 4, we study the basic topological properties of our topological spaces of persistence modules. Throughout, most of our arguments are elementary, except our proof of completeness which uses basic ideas from category theory. We also provide an appendix where we examine interleavings of interval modules.

\section{BACKGROUND}

In this section we define persistence modules and interleaving distance, giving examples and basic properties. We also define extended pseudometric spaces and their induced topological spaces.

2.1. Persistence modules Let $\mathbf{k}$ be a fixed field. A persistence module $M$ is a set of $\mathbf{k}$ vector spaces $\{M(a) \mid a \in \mathbb{R}\}$ together with k-linear maps $\left\{v_{a}^{b}: M(a) \rightarrow M(b) \mid a \leq b\right\}$ such that

i): for all $a, v_{a}^{a}: M(a) \rightarrow M(a)$ is the identity map, and

ii): if $a \leq b \leq c$ then $v_{a}^{c}=v_{b}^{c} \circ v_{a}^{b}$.

Equivalently, a persistence module is a functor $M: \underline{\mathbf{R}} \rightarrow \underline{\operatorname{Vect}}_{\mathbf{k}}$, where $\underline{\mathbf{R}}$ is the category whose set of objects is $\mathbb{R}$ and whose morphisms are the inequalities $a \leq b$, and $\underline{\text { Vect }}_{\mathbf{k}}$ is the category of $\mathbf{k}$-vector spaces and $\mathbf{k}$-linear maps.

Example 2.1. Let $X$ be a topological space and $f: X \rightarrow \mathbb{R}$ be a function. For each $a \in \mathbb{R}$ the subset

$$
F_{a}:=\{x \in X \mid f(x) \leq a\} \subset X
$$

is called a sublevel set. Note that $a \leq b$ implies $F_{a} \subset F_{b}$ so that we have an inclusion map $i_{a}^{b}: F_{a} \hookrightarrow F_{b}$ for all $a \leq b$. This inclusion map induces a linear map

$$
H_{n}\left(i_{a}^{b}\right): H_{n}\left(F_{a} ; \mathbf{k}\right) \rightarrow H_{n}\left(F_{b} ; \mathbf{k}\right)
$$

on singular homology groups with a coefficients in $\mathbf{k}$ of degree $n \geq 0$. We thus have a persistence module $H F: \underline{\mathbf{R}} \rightarrow \underline{\text { Vect }}_{\mathbf{k}}$ given by $H F(a)=H_{n}\left(F_{a} ; \mathbf{k}\right)$ and $H F(a \leq b)=H_{n}\left(i_{a}^{b}\right)$.

Example 2.2. Consider the half open interval $[0,2)$ in $\mathbb{R}$ and define the persistence module $\chi: \underline{\mathbf{R}} \rightarrow \underline{\text { Vect }}_{\mathbf{k}}$ given by

$$
\chi(a)=\left\{\begin{array}{ll}
\mathbf{k} & a \in[0,2) \\
0 & \text { otherwise }
\end{array} \quad \text { and } \quad \chi(a \leq b)= \begin{cases}1 & a, b \in[0,2) \\
0 & \text { otherwise }\end{cases}\right.
$$

where 1 is the identity map on $\mathbf{k}$. For simplicity, we will abuse notation and denote this persistence module by $[0,2)$.

Example 2.3. Replacing $[0,2)$ in the above with an arbitrary interval $J \subset \mathbb{R}$ we obtain a persistence module that we call an interval module and we will also denote by $J$. 
Example 2.4. A trivial but important example is the zero module, denoted 0, that has $0(a)=0$ for all $a$.

A morphism of persistence modules $M$ and $N$ is a collection of linear maps $\left\{\varphi_{a}: M(a) \rightarrow\right.$ $N(a) \mid a \in \mathbb{R}\}$ such that the following diagram commutes for each pair $a \leq b$.

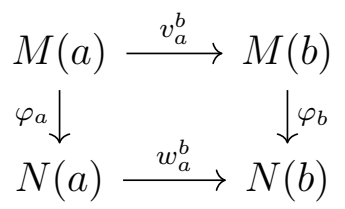

Equivalently, a morphism of persistence modules is a natural transformation $\varphi: M \Rightarrow N$. We will often denote a morphism of persistence modules as $\varphi: M \rightarrow N$. Such a morphism is an isomorphism if and only if each linear map $\varphi_{a}$ is an isomorphism.

Example 2.6. It is a good exercise to check that because of the constraints due to the commutative squares in (2.5), there is a nonzero morphism from the interval module $[a, b)$ to the interval module $[c, d)$ only if $c \leq a \leq d \leq b$.

In the appendix, we present a more thorough discussion of interval modules (Section A.1) and maps between them (Section A.2).

2.2. Indecomposables Given two persistence modules $M$ and $N$, their direct sum is the persistence module $M \oplus N$ given by $(M \oplus N)(a)=M(a) \oplus N(a)$ and $(M \oplus N)(a \leq b)=$ $M(a \leq b) \oplus N(a \leq b)$. In the same way we can define the direct sum of a collection of persistence modules indexed by an arbitrary set.

A persistence module is said to be indecomposable if it is not isomorphic to a nontrivial direct sum. For example, interval modules are indecomposable. However, not all indecomposable persistence modules are interval modules (see [18, Theorem 2.5, Remark 2.6] for a discussion of examples due to do Webb [42], Lesnick, and Crawley-Boevey).

A special case of the following theorem follows from work of Gabriel [28], but the general case was proved by Crawley-Boevey [23].

Theorem 2.7 (Structure Theorem). Let $M: \underline{\mathbf{R}} \rightarrow \underline{\text { Vect }}_{\mathbf{k}}$ be a persistence module. If $M(a)$ is finite dimensional for each $a \in \mathbb{R}$, then $M$ is isomorphic to a direct sum of interval modules.

2.3. Interleaving distance Interleaving distance was introduced in [15] and further studied in the context of multiparameter persistence in [31. Here we also adopt the categorical point of view from [12].

Definition 2.8. Let $\varepsilon \geq 0$. An $\varepsilon$-interleaving between persistence modules $M$ and $N$ consists of morphisms $\varphi_{a}: M(a) \rightarrow N(a+\varepsilon)$ and $\psi_{a}: N(a) \rightarrow M(a+\varepsilon)$ for all $a$ such that the following four diagrams commute for all $a \leq b$, where the horizontal maps are given by the respective persistence modules.
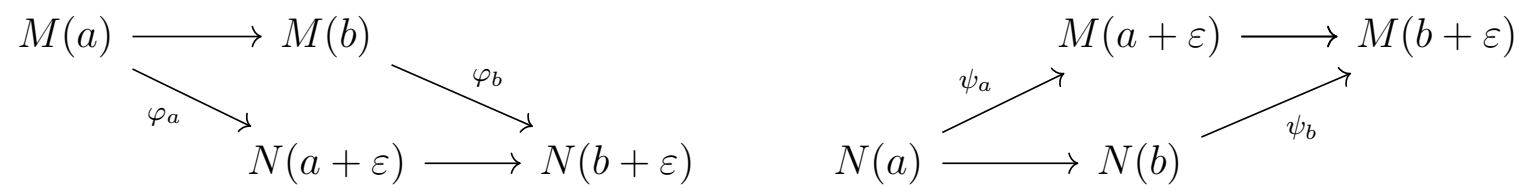

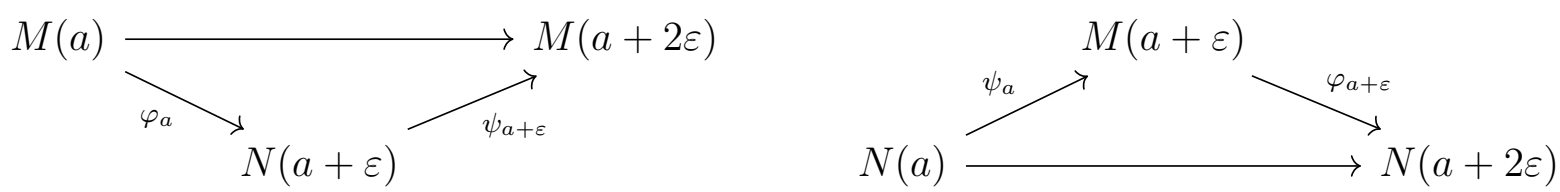

Equivalently, we may describe this in terms of natural transformations. First, for $x \in \mathbb{R}$ let $T_{x}: \underline{\mathbf{R}} \rightarrow \underline{\mathbf{R}}$ denote the functor given by $T_{x}(a)=a+x$. Next if $x \geq 0$, let $\eta_{x}: 1_{\underline{\mathbf{R}}} \Rightarrow T_{x}$ denote the natural transformation from the identity functor on $\underline{\mathbf{R}}$ to $T_{x}$ that has components $\left(\eta_{x}\right)_{a}: a \leq a+x$. Then an $\varepsilon$-interleaving consists of natural transformations $\varphi: M \Rightarrow N T_{\varepsilon}$ and $\psi: N \Rightarrow M T_{\varepsilon}$ such that $\left(\psi T_{\varepsilon}\right) \varphi=M \eta_{2 \varepsilon}$ and $\left(\varphi T_{\varepsilon}\right) \psi=N \eta_{2 \varepsilon}$. See [12, Section 3] for more details. We say $M$ and $N$ are $\varepsilon$-interleaved.

Remark 2.11. Two persistence modules are 0-interleaved if and only if they are isomorphic. If persistence modules $M$ and $N$ are $\varepsilon$-interleaved and $N$ and $P$ are $\delta$-interleaved then $M$ and $P$ are $(\varepsilon+\delta)$-interleaved.

Definition 2.12. Let $M$ and $N$ be two persistence modules. Then the interleaving distance $d_{I}(M, N)$ between $M$ and $N$ is defined as

$$
d_{I}(M, N):=\inf (\varepsilon \in[0, \infty) \mid M \text { and } N \text { are } \varepsilon \text {-interleaved })
$$

If no such $\varepsilon$ exists, then $d_{I}(M, N)=\infty$.

Example 2.13. The interval modules $[0,2]$ and $(0,2)$ are not 0 -interleaved. In fact, there are no nonzero maps between $[0,2]$ and $(0,2)$. However they are $\varepsilon$-interleaved for all $\varepsilon>0$. Thus, $d_{I}([0,2],(0,2))=0$.

Example 2.14. The interval modules $M=[0,1)$ and $N=[0, \infty)$ are not $\varepsilon$-interleaved for any $\varepsilon \geq 0$. Indeed, assume $\varphi$ and $\psi$ provide such an interleaving. Consider the following trapezoid.

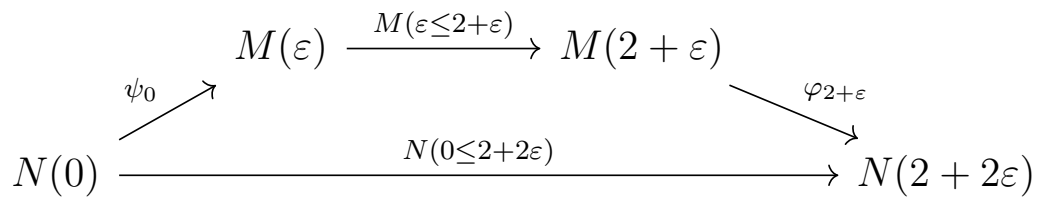

It decomposes into a commutative parallelogram and commutative triangle from (2.9) and (2.10) in two different ways. In either case, this diagram commutes. Furthermore, the bottom horizontal arrow is the identity on $\mathbf{k}$ and the top horizontal arrow is 0 , which is a contradiction.

In the appendix, we give a careful study of interleavings of interval modules (Section A.3). We will make use of the following lemma without reference.

Lemma 2.15 (Converse Algebraic Stability Theorem [31, Theorem 3.4]). Let $\varepsilon \geq 0$. If for all $\alpha \in A$, the persistence modules $I_{\alpha}$ and $J_{\alpha}$ are $\varepsilon$-interleaved, then $\bigoplus_{\alpha \in A} I_{\alpha}$ and $\bigoplus_{\alpha \in A} J_{\alpha}$ are $\varepsilon$-interleaved. Thus $d_{I}\left(\bigoplus_{\alpha \in A} I_{\alpha}, \bigoplus_{\alpha \in A} J_{\alpha}\right) \leq \sup _{\alpha \in A} d_{I}\left(I_{\alpha}, J_{\alpha}\right)$.

Proof For $\alpha \in A$, let $\varphi_{\alpha}$ and $\psi_{\alpha}$ be maps giving an $\varepsilon$-interleaving of $I_{\alpha}$ and $J_{\alpha}$. Then $\bigoplus \varphi_{\alpha}$ and $\bigoplus \psi_{\alpha}$ provide the desired $\varepsilon$-interleaving. 


\subsection{Pseudometric spaces}

Definition 2.16. A pseudometric on a set $X$ is a map $d: X \times X \rightarrow[0, \infty)$ that satisfies

M1): $d(x, x)=0$,

M2): $d(x, y)=d(y, x)$, and

M3): $d(x, y) \leq d(x, z)+d(z, y)$

for all $x, y, z \in X$. Note that we have omitted the condition $d(x, y)=0$ implies $x=y$ required of metric. More generally, an extended pseudometric on $X$ is a map $d: X \times X \rightarrow$ $[0, \infty]$ satisfying the same three axioms. We call a set with an (extended) pseudometric an (extended) pseudometric space.

Theorem 2.17 ([15, 31, 12]). The interleaving distance is an extended pseudometric on any set of (isomorphism classes of) persistence modules.

Remark 2.18. A proper class of persistence modules with the interleaving distance is not an extended pseudometric space since it is not a set. However it is a symmetric Lawvere space [9, 8, 10].

In an extended (pseudo)metric space, the condition $d(x, y)<\infty$ defines an equivalence relation. As a result, such a space has a natural partition into (pseudo)metric spaces.

In an (extended) pseudometric space one can consider equivalence classes of the equivalence relation $x \sim y$ if $d(x, y)=0$ to obtain an (extended) metric space. However, for persistence modules, one may be interested in distinguishing nonisomorphic modules with zero interleaving distance, so we will not apply this simplification.

Any extended pseudometric on a set induces a topology on it. Indeed, for any $x \in X$ and a real number $r>0$ consider the open ball $B_{r}(x)$ centered at $x$ with radius $r$,

$$
B_{r}(x):=\{y \in X \mid d(x, y)<r\} .
$$

We call a set $O$ open in $X$ if for each $x \in O$, there exists $r>0$ such that $B_{r}(x) \subset O$. Then it is easy to check that the collection of all open sets is a topology on $X$.

Note that each open ball $B_{r}(x)$ is also an open set in $X$ and the collection of all open balls forms a base for this topology $X$ since each open set $O$ in $X$ can be written as a union of open balls.

Example 2.19. Consider the interval module $[0,5)$ and let $\varepsilon>1$. Then the ball $B_{\varepsilon}([0,5))$ contains the interval modules $[-1,6]$ and $(1,4)$.

In the appendix, we study the interval modules in an $\varepsilon$-neighborhood of an interval module (Section A.4).

A sequence $\left(x_{n}\right)_{n \geq 1}$ in an extended pseudometric space $X$ is said to converge to $x \in X$ if for all $\varepsilon>0$ there exists $N>0$ such that for all $n \geq N, d\left(x_{n}, x\right)<\varepsilon$. The point $x$ is called a limit of the sequence. Note that in an extended pseudometric space we no longer have unique limits, but we do have that if $x$ and $x^{\prime}$ are limits, then by the triangle inequality $d\left(x, x^{\prime}\right)=0$.

A sequence $\left(x_{n}\right)_{n \geq 1}$ in an extended pseudometric space is a Cauchy sequence if for all $\varepsilon>0$ there exists an $N>0$ such that for all $n, m \geq N, d\left(x_{n}, x_{m}\right)<\varepsilon$. If a subsequence of a Cauchy sequence has a limit $x$, then by the triangle inequality, $x$ is also a limit of the Cauchy sequence. 


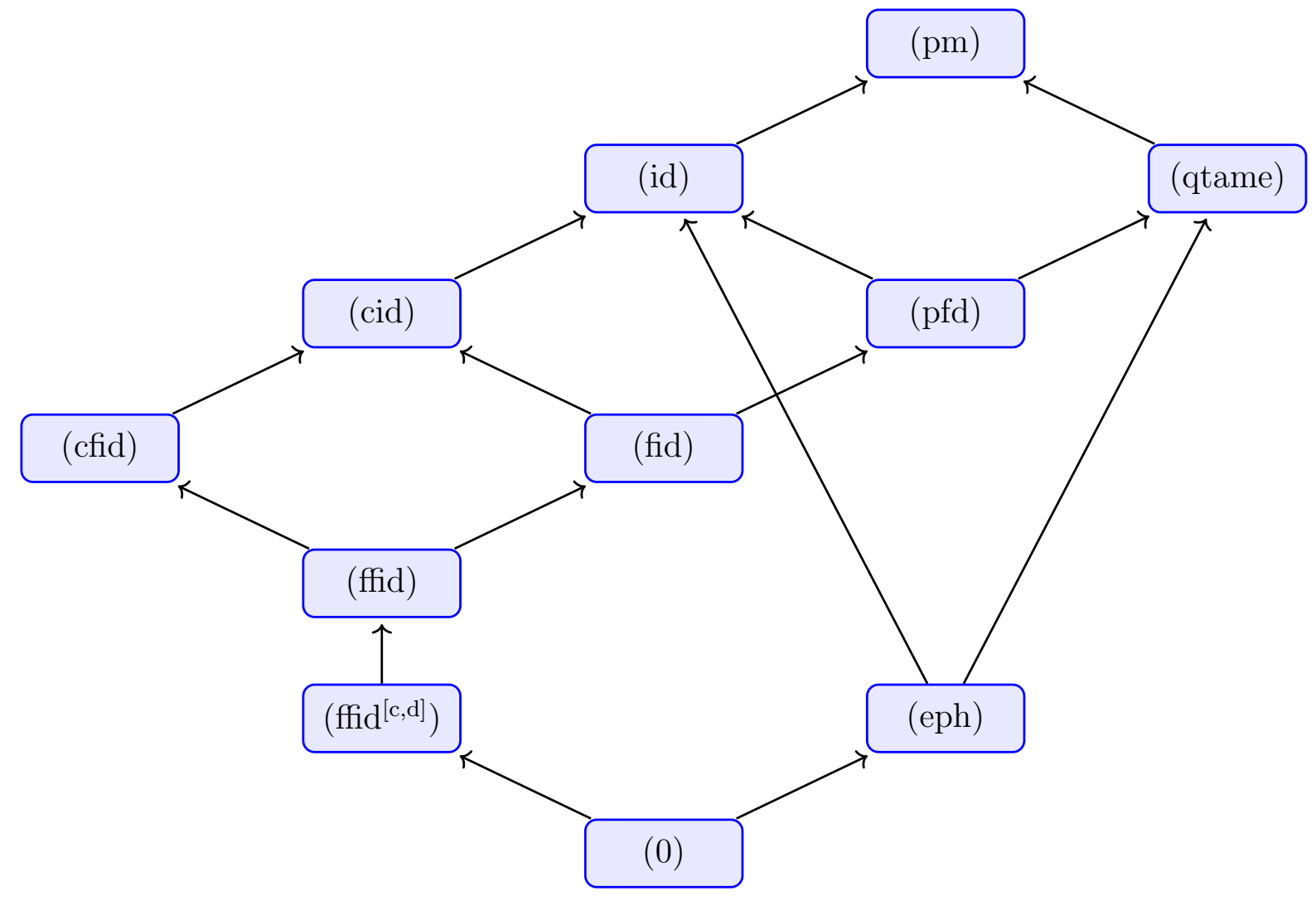

Figure 1. Hasse diagram of sets and classes of persistence modules.

\section{Sets AND Classes of PERSistenCE MOdUleS}

In this section we define classes of persistence modules that contain many of the persistence modules considered in the literature. We study the relationships between these classes and determine which of them are in fact sets.

For the remainder of the paper, we will only consider isomorphism classes of persistence modules. That is, whenever we say 'persistence module', we really mean 'isomorphism class of persistence modules'. This is standard when discussing both vector spaces and persistence modules.

3.1. Classes of persistence modules In this section, we consider the classes of persistence modules in Figure 1, which we now describe.

- (pm) is the class of persistence modules.

- (id) is the class of interval-decomposable persistence modules: those isomorphic to $\bigoplus_{\alpha \in A} I_{\alpha}$, where $A$ is some indexing set, and each $I_{\alpha}$ is an interval module.

- (cid), the countably interval-decomposable persistence modules, is the subclass of (id) where the index set $A$ is countable.

- (cfid), the countably finite-interval decomposable persistence modules, is the subclass of (cid) in which each interval $I_{\alpha}$ is finite.

- (fid), the finitely interval-decomposable persistence modules, is the class of persistence modules isomorphic to $\bigoplus_{k=1}^{N} I_{k}$ for some $N$, where each $I_{k}$ is an interval module. 
- (ffid), the finitely finite-interval decomposable persistence modules, is the subclass of (fid) in which each $I_{k}$ is a finite interval.

- Given $c<d$, (ffid ${ }^{[\mathrm{c}, \mathrm{d}]}$ ) is the subclass of (ffid) in which each $I_{k} \subset[c, d]$.

- (pfd), the pointwise finite dimensional persistence modules, is the class of all persistence modules $M$ with each $M(a)$ finite dimensional.

- (qtame), the q-tame persistence modules, is the class of all persistence modules $M$ where each $a<b$ the linear map $v_{a}^{b}: M(a) \rightarrow M(b)$ has a finite rank.

- (eph), the ephemeral persistence modules, is the class of all persistence modules $M$ where for each $a<b$ the linear map $v_{a}^{b}: M(a) \rightarrow M(b)$ is zero.

- (0) is the class consisting of only the zero persistence module.

Remark 3.1. The class (fid) is a slight generalization of the class of constructible persistence modules. A persistence module $M$ is said to be constructible [37] if there exists a finite subset $A=\left\{a_{1}, \ldots, a_{n}\right\}$ of $\mathbb{R}$ such that

- for $t<a_{1}, M(t)=0$,

- for $a_{i} \leq s \leq t<a_{i+1}, M(s \leq t)$ is an isomorphism where $i \in\{1, \ldots, n-1\}$, and

- for $a_{n} \leq s \leq t, M(s \leq t)$ is an isomorphism.

A constructible module $M$, satisfies $M \cong \bigoplus_{k=1}^{N} I_{k}$ where each $I_{k}$ is of the form $\left[a_{i}, a_{j}\right)$ or $\left[a_{i}, \infty\right)[1]$

\subsection{Inclusions}

Lemma 3.2. Let $M$ be an ephemeral module. Then $M \cong \bigoplus_{\alpha \in A} M_{\alpha}$, where each $M_{\alpha} \cong[r, r]$ for some $r \in \mathbb{R}$.

Proof Let $M \in(\mathrm{eph})$. For $r \in \mathbb{R}$, let $M_{r}$ be the persistence module with $M_{r}(x)=M(r)$ if $x=r$ and otherwise $M_{r}(x)=0$. Then $M \cong \oplus_{r \in \mathbb{R}} M_{r}$. Furthermore each $M(r)$ has a basis, so $M_{r}$ decomposes over this basis into $[r, r]$ interval modules.

Proposition 3.3. The diagram in Figure 1 is a Hasse diagram for the poset structure of these classes of persistence modules under the inclusion order.

Proof By Theorem 2.7, (pfd) is in (id). By Lemma 3.2, (eph) $\subset$ (id). It is easy to check that all of the other arrows indicated in the diagram are inclusions and that in fact all of the inclusions are proper. With the observation that if $A \subset B, C \subset D$ and $A \not \subset D$ then $B \not \subset C$, it remains to check the following cases.

(1) (eph) $\not \subset(\mathrm{pfd}): \bigoplus_{k=1}^{\infty}[0,0]$ is in (eph) but not in (pfd).

(2) (eph) $\not \subset$ (cid): $\bigoplus_{r \in \mathbb{R}}[0,0]$ is in (eph) but not in (cid).

(3) (ffid $\left.^{[\mathrm{c}, \mathrm{d}]}\right) \not \subset(\mathrm{eph}):[c, d]$ is in (ffid $\left.{ }^{[\mathrm{c}, \mathrm{d}]}\right)$ but is not in (eph).

(4) (fid) $\not \subset$ (cfid): $[0, \infty)$ is in (fid) but is not in (cfid).

(5) (pfd) $\not \subset$ (cid): $\bigoplus_{r \in \mathbb{R}}[r, r]$ is in (pfd) but is not in (cid).

(6) (cfid) $\not \subset$ (qtame): $\bigoplus_{k=1}^{\infty}[0,1)$ is in (cfid) but is not in (qtame).

(7) (qtame) $\not \subset(\mathrm{id}): \prod_{k=1}^{\infty}\left[0, \frac{1}{k}\right)$ is in (qtame) but is not in (id) [17].

\footnotetext{
${ }^{1}$ In particular, the multiplicity of $\left[a_{i}, a_{j}\right)$ can be calculated using the inclusion/exclusion formula $\operatorname{rank} M\left(a_{i} \leq a_{j-1}\right)-\operatorname{rank} M\left(a_{i} \leq a_{j}\right)-\operatorname{rank} M\left(a_{i-1} \leq a_{j-1}\right)+\operatorname{rank} M\left(a_{i-1} \leq a_{j}\right)$ [21], which is an example of Möbius inversion [37.
} 


\subsection{Almost inclusions}

Definition 3.4. Say that a class of persistence modules $\mathcal{A}$ almost includes in a class of persistence modules $\mathcal{B}$ if for each $A \in \mathcal{A}$ there exists an element $B \in \mathcal{B}$ such that $d_{I}(A, B)=$ 0 .

Lemma 3.5. A finite sequence of inclusions and almost inclusions is an almost inclusion.

Proof This follows from the triangle inequality.

Lemma 3.6. $M$ is an ephemeral persistence module if and only if $d_{I}(M, 0)=0$. That is, (eph) almost includes in (0).

Proof Let $M$ be an ephemeral persistence module. Then $M$ and 0 are $\varepsilon$-interleaved for all $\varepsilon>0$ by the zero maps.

Next assume $d_{I}(M, 0)=0$. Consider $a<b$. Let $\varepsilon=\frac{b-a}{2}$. Since $M$ and 0 are $\varepsilon$-interleaved, the map $M(a<b)$ factors through 0 , and is thus the zero map.

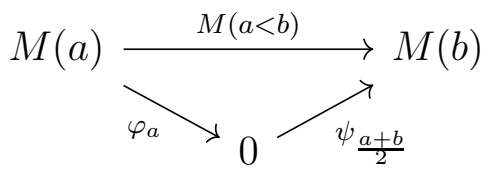

Therefore $M$ is an ephemeral persistence module.

For a persistence module $M$, define the radical of $M$ by $(\operatorname{rad} M)(a)=\sum_{c<a} \operatorname{im} M(c<$ a) [17]. Note that $\operatorname{rad} M \subset M$ and inherits the structure of a persistence module.

Proposition 3.7. Let $M$ be a persistence module. Then $d_{I}(M, \operatorname{rad} M)=0$.

Proof Let $\varepsilon>0$. For all $a \in \mathbb{R}$, let $\varphi_{a}=M(a<a+\varepsilon):(\operatorname{rad} M)(a) \rightarrow M(a+\varepsilon)$, and let $\psi_{a}=M(a<a+\varepsilon): M(a) \rightarrow(\operatorname{rad} M)(a+\varepsilon)$. Then by the functoriality of $M$, this is an $\varepsilon$-interleaving of $\operatorname{rad} M$ and $M$. Therefore $d_{I}(\operatorname{rad} M, M)=0$.

Theorem 3.8. Let $M \in$ (qtame). Then $\operatorname{rad} M \in$ (qtame) and $\operatorname{rad} M \in$ (cid).

Proof Let $M \in$ (qtame). Since $\operatorname{rad} M$ is a submodule of $M$, it follows that $\operatorname{rad} M \in$ (qtame) as well. By [17, Corollary 3.6], $\operatorname{rad} M \in(\mathrm{id})$. We will strengthen this to show that $\operatorname{rad} M \in$ (cid).

Since $\operatorname{rad} M \in(\mathrm{id}), \operatorname{rad} M \cong \bigoplus_{\alpha \in A} I_{\alpha}$. For $q, r \in \mathbb{Q}$ with $q<r$, let $A_{q, r}=\{\alpha \in A$ $\left.q, r \in I_{\alpha}\right\}$, and let $A^{\prime}=\bigcup_{q<r \in \mathbb{Q}} A_{q, r}$. Since $\operatorname{rad} M \in$ (qtame), for each $q<r \in \mathbb{Q},\left|A_{q, r}\right|<\infty$. Therefore $A^{\prime}$ is countable.

Furthermore, by definition, for each $a \in \mathbb{R}$ and for each $x \in(\operatorname{rad} M)(a)$ there exists $c<a$ and $y \in M(c)$ such that $M(c \leq a)(y)=x$. Choose $b \in(c, a)$. Then $z:=M(c \leq b)(y) \in$ $(\operatorname{rad} M)(b)$ and $(\operatorname{rad} M)(b \leq a)(z)=x$. Hence the interval decomposition of $\operatorname{rad} M$ does not contain any one-point intervals, and thus $A=A^{\prime}$. Therefore $\operatorname{rad} M \in(\mathrm{cid})$.

Combining the previous two results we have the following.

Corollary 3.9. Let $M \in$ (qtame). Then there exists $N \in\left(\right.$ cid) such that $d_{I}(M, N)=0$. That is, (qtame) almost includes in (cid). 
3.4. Enveloping distance In this section, we define a non-symmetric distance between classes of persistence modules and calculate its value for most of the pairs in Figure 1.

Definition 3.10. Let $\mathcal{A}$ and $\mathcal{B}$ be classes of persistence modules. We define the enveloping distance from $\mathcal{A}$ to $\mathcal{B}$ as follows.

$$
E(\mathcal{A}, \mathcal{B})=\inf (r \quad \mid \forall B \in \mathcal{B} \text { and } s>r, \exists A \in \mathcal{A} \text { such that } A, B \text { are } s \text {-interleaved })
$$

If there is no such $r$, we set $E(\mathcal{A}, \mathcal{B})=\infty$.

For example, as we will demonstrate later in this section, $E\left((0),\left(\mathrm{ffid}^{[\mathrm{c}, \mathrm{d}]}\right)\right)=\frac{d-c}{2}$ and $E\left(\left(\right.\right.$ ffid $\left.\left.^{[\mathrm{c}, \mathrm{d}]}\right),(0)\right)=0$.

We will use the following basic fact about interleavings.

Lemma 3.11 ([31, 12]). If persistence modules $A$ and $B$ are s-interleaved and persistence modules $B$ and $C$ are $t$-interleaved, then $A$ and $C$ are $(s+t)$-interleaved.

The enveloping distance has the following properties.

Lemma 3.12. $E(\mathcal{A}, \mathcal{A})=0$ and $E(\mathcal{A}, \mathcal{C}) \leq E(\mathcal{A}, \mathcal{B})+E(\mathcal{B}, \mathcal{C})$.

Proof For reflexivity, each persistence module is $s$-interleaved with itself for all $s \geq 0$. The triangle inequality follows from Lemma 3.11 .

Definition 3.13. In the case that $E(\mathcal{A}, \mathcal{B})=\infty$, we write that $E(\mathcal{A}, \mathcal{B})=\infty^{-}$if $\forall B \in \mathcal{B} \exists s$ and $A \in \mathcal{A}$ such that $A, B$ are $s$-interleaved. From now on we reserve $E(\mathcal{A}, \mathcal{B})=\infty$ for the case that this condition is not satisfied.

Lemma 3.14. If $\mathcal{A}$ (almost) includes in $\mathcal{B}$ then $E(\mathcal{B}, \mathcal{A})=0$.

Proof This follows immediately from the definitions.

Corollary 3.15. $E((0),(\mathrm{eph}))=0$ and $E((\mathrm{eph}),(0))=0$.

Lemma 3.16. If $\mathcal{A}$ (almost) includes in $\mathcal{B}, E(\mathcal{B}, \mathcal{C})=\infty$, and $\mathcal{C}$ (almost) includes in $\mathcal{D}$, then $E(\mathcal{A}, \mathcal{D})=\infty$.

Proof Assume $E(\mathcal{A}, \mathcal{D})<\infty$. Then there is some $s \geq 0$ such that for all $D \in \mathcal{D}$ there exists an $A \in \mathcal{A}$ such that $D$ and $A$ are $s$-interleaved.

Let $\varepsilon>0$. Let $C \in \mathcal{C}$. Since $\mathcal{C}$ (almost) includes in $\mathcal{D}$, there is a $D \in \mathcal{D}$ such that $C$ and $D$ are $\varepsilon$-interleaved. By our first observation, there is an $A \in \mathcal{A}$ such that $D$ and $A$ are $s$-interleaved. Since $\mathcal{A}$ (almost) includes in $\mathcal{B}$, there is a $B \in \mathcal{B}$ such that $A$ and $B$ are $\varepsilon$-interleaved. Therefore by Remark 2.11, $C$ and $B$ are $(s+2 \varepsilon)$-interleaved. So for all $C \in \mathcal{C}$ there is a $B \in B$ such that $C$ and $B$ are $(s+2 \varepsilon)$-interleaved. Thus $E(\mathcal{B}, \mathcal{C})<\infty$.

Proposition 3.17. (1) We have the following enveloping distances: $E\left((0),\left(\mathrm{ffid}^{[\mathrm{c}, \mathrm{d}]}\right)\right)=$ $\frac{d-c}{2}$ and $E\left(\left(\right.\right.$ ffid $\left.{ }^{[\mathrm{c}, \mathrm{d}]}\right),($ ffid $\left.)\right)=\infty^{-}$. Also, $E((0),($ ffid $))=\infty^{-}, E((\mathrm{eph}),($ ffid $))=\infty^{-}$ and $E\left((\right.$ eph $),\left(\right.$ ffid $\left.\left.^{[\mathrm{c}, \mathrm{d}]}\right)\right)=\frac{d-c}{2}$.

(2) In addition, $E$ ((cfid), (fid) $)=\infty$ and $E$ ((qtame), (cfid) $)=\infty$.

(3) With the exception of $(0) \subset($ eph $),(0) \subset$ (ffid $\left.^{[\mathrm{c}, \mathrm{d}]}\right)$, (ffid $\left.{ }^{[\mathrm{c}, \mathrm{d}]}\right) \subset$ (ffid) and the possible exception of $(\mathrm{pfd}) \subset($ qtame), all of the other inclusions $\mathcal{A} \subset \mathcal{B}$ in Figure 11 have enveloping distance $E(\mathcal{A}, \mathcal{B})=\infty$. Also $E(($ qtame $),($ cid $))=\infty$. 
Proof $\quad(1) \quad-(0) \subset\left(\right.$ ffid $\left.^{[c, \mathrm{~d}]}\right): d_{I}([c, d], 0)=\frac{d-c}{2}$ and for all $M \in\left(\right.$ ffid $\left.^{[c, \mathrm{~d}]}\right), d_{I}(M, 0) \leq$ $\frac{d-c}{2}$.

- (ffid $\left.{ }^{[c, \mathrm{~d}]}\right) \subset$ (ffid): For all $M \in\left(\right.$ ffid $\left.{ }^{[\mathrm{c}, \mathrm{d}]}\right)$ and $N \in$ (ffid), $d_{I}(M, N) \leq d_{I}(M, 0)+$ $d_{I}(0, N)<\infty$. Let $z \geq 0$. For all $M \in\left(\right.$ ffid $\left.^{[c, d]}\right)$, there are no nontrivial maps from $M$ to $(d, d+2 z]$. Thus $d_{I}(M,(d, d+2 z]) \geq d_{I}((d, d+2 z], 0) \geq z$.

- The other three cases follow from the same arguments.

- (cfid) to (fid): Consider $[0, \infty)$.

- (qtame) to (cfid): Consider $\bigoplus_{k=1}^{\infty}[0, k)$.

- (ffid) $\subset$ (fid): Consider $[0, \infty)$.

- (ffid) $\subset$ (cfid): Consider $\bigoplus_{k=1}^{\infty}[0, k)$.

- (cfid) $\subset$ (cid): Consider $[0, \infty)$.

- (fid) $\subset$ (cid): Consider $\bigoplus_{k=1}^{\infty}[0, \infty)$.

- (fid) $\subset\left(\right.$ pfd): Consider $\bigoplus_{k=0}^{\infty}\left[2^{k}, 2^{k+1}\right)$.

- (cid) $\subset$ (id): Consider $\bigoplus_{r \in \mathbb{R}}[0, \infty)$.

- (id) $\subset$ (pm): Consider $\prod_{k=1}^{\infty}[0, \infty)$.

- $($ eph $) \subset($ id $),($ eph $) \subset($ qtame $),($ pfd $) \subset($ id $)$, (qtame $) \subset($ cid $)$, and (qtame $) \subset$ (pm) follow from Lemma 3.16.

Remark 3.18. Together with Corollary 3.15, Lemma 3.14, and Lemma 3.16, this proposition implies all of the pairwise enveloping distances between the sets and classes of persistence modules in Figure 2, except $E((\mathrm{pfd}),($ qtame $))$. For example, $E((\mathrm{id})$, (qtame) $)=0$, $E((\mathrm{cid}),(\mathrm{pfd}))=0$, and $E((\mathrm{cid}),($ qtame $))=0$ by Lemmas 3.14 and 3.5, and $E(($ fid $),(\mathrm{cfid}))=$ $\infty$ by Lemma 3.16 .

We end this section by showing that $E(($ pfd $),($ qtame $))=0$. First we give a definition.

Definition 3.19. Let $M$ be a persistence module. Let $p \geq 0$. We define the $p$-persistent submodule of $M$ by

$$
M^{(p)}(a)=\operatorname{im} M(a-p \leq a) .
$$

For $a \leq b$, there is an induced map between objects $M^{(p)}(a)$ and $M^{(p)}(b)$ given by $M(a \leq b)$. Since $M$ is a persistence module, so is $M^{(p)}$, and since $M^{(p)}(a)$ is a sub-vector space of $M(a)$ for all $a, M^{(p)}$ is a submodule of $M$.

Proposition 3.20. Let $M$ be a persistence module and let $p \geq 0$. Then $M$ and $M^{(p)}$ are p-interleaved.

Proof For $a \in \underline{\mathbf{R}}$, define $\varphi_{a}: M(a) \rightarrow M^{(p)}(a+p)$ by $\varphi_{a}=M(a \leq a+p)$, and $\psi_{a}: M^{(p)}(a) \rightarrow$ $M(a+p)$ by $\psi_{a}=M(a \leq a+p)$. Then all the arrows in diagrams (2.9) and (2.10) are maps in $M$ and hence commute.

Corollary 3.21. $E((\mathrm{pfd}),($ qtame $))=0$.

Proof Let $M$ be a q-tame persistence module. Let $p>0$. Then by definition, $M^{(p)}$ is a pointwise finite-dimensional persistence module. By Proposition [3.20, $M$ and $M^{(p)}$ are $p$-interleaved. Thus, by definition, $E(($ pfd $),($ qtame $))=0$. 


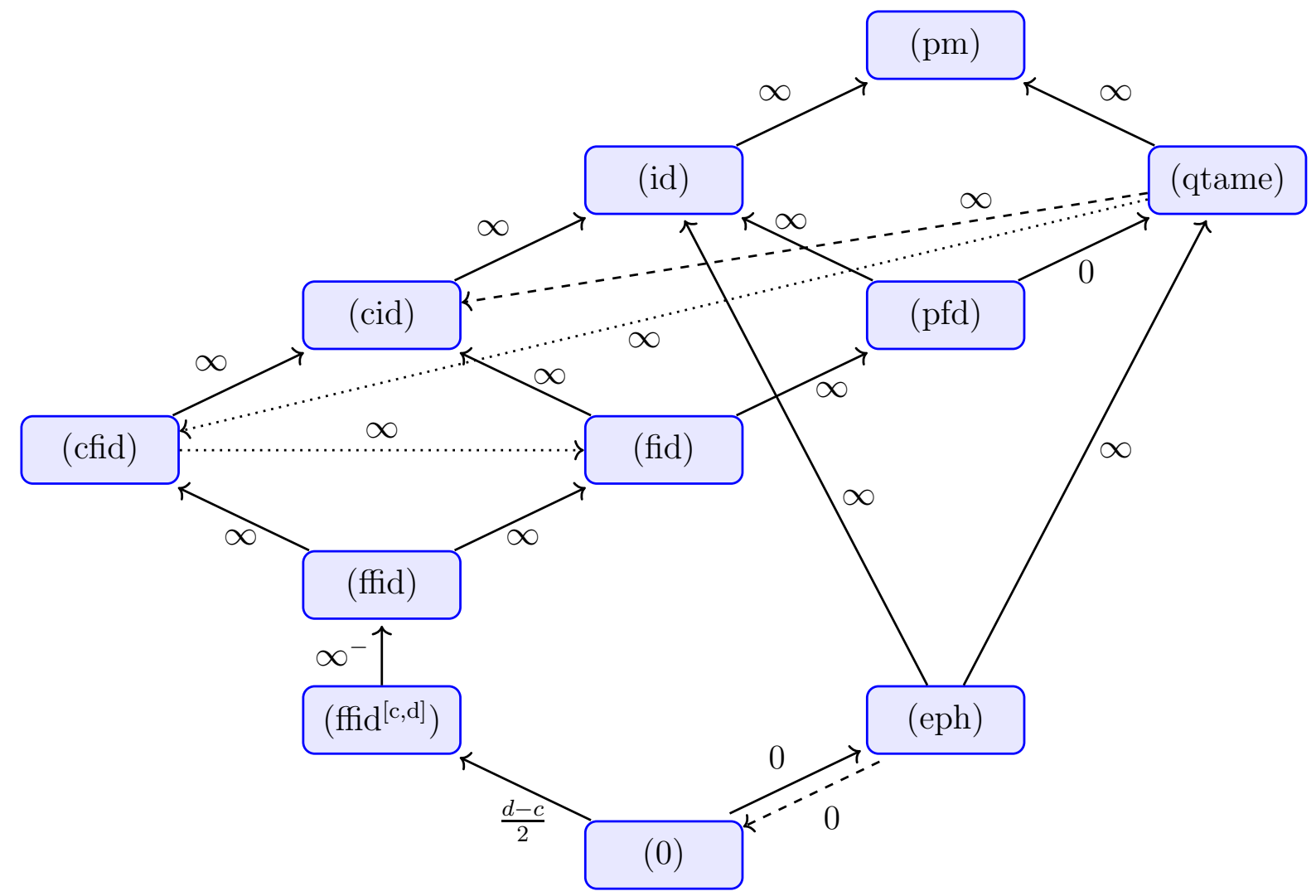

Figure 2. Diagram of sets and classes of persistence modules. Solid arrows indicate inclusions, dashed arrows indicate almost inclusions, and dotted arrows do not indicate any relationship. Annotations of arrows indicate enveloping distance from the source to the target, given in Definitions 3.10 and 3.13 .

3.5. Sets of persistence modules Next we consider whether the classes defined above are sets or proper classes. We will use the following notation. Let $\overline{\mathbb{R}}:=\mathbb{R} \cup\{ \pm \infty\}$ and $\overline{\mathbb{N}}:=\mathbb{N} \cup\{\infty\}$. Given a set $X$, let $\mathcal{P}(X)$ denote its power set. Let $\mathbb{I}$ be the set of all intervals in $\mathbb{R}$. We define a map, $f: \mathbb{I} \longrightarrow\{1,2,3,4\}$ by

$$
f(I)= \begin{cases}1, & \inf I \notin I, \sup I \notin I \\ 2, & \inf I \in I, \sup I \notin I \\ 3, & \inf I \notin I, \sup I \in I \\ 4, & \inf I \in I, \sup I \in I .\end{cases}
$$

Proposition 3.22. The class (cid) is a set.

Proof Consider the map

$$
(\text { cid }) \longrightarrow \mathcal{P}\left(\overline{\mathbb{R}}^{2} \times\{1,2,3,4\} \times \overline{\mathbb{N}}\right)
$$

defined by

$$
\bigoplus_{\alpha \in A} I_{\alpha} \longmapsto \bigcup_{\alpha \in A}\left[\left\{\left(\inf I_{\alpha}, \sup I_{\alpha}\right)\right\} \times\left\{f\left(I_{\alpha}\right)\right\} \times\{m(i)\}\right]
$$


where $m(i)$ denotes the multiplicity of the direct summand $I_{\alpha}$. This map is an injection, hence (cid) is a set.

Corollary 3.23. Therefore the classes (cfid), (fid), (ffid), (ffid ${ }^{[\mathrm{c}, \mathrm{d}]}$ ), and (0) are also sets.

Lemma 3.24. Each interval appears only finitely many times in the direct-sum intervalmodule decomposition of a pointwise finite-dimensional persistence module.

Proof For each interval $I, \bigoplus_{k=1}^{\infty} I \notin(\mathrm{pfd})$.

Proposition 3.25. The class (pfd) is a set.

Proof Let $M \in(\mathrm{pfd})$. By Theorem 2.7, $M \cong \bigoplus_{\alpha \in A} I_{\alpha}$ where $I_{\alpha}$ is an interval and $A$ is a set. By Lemma 3.24, we can define the following map.

$$
\begin{gathered}
(\operatorname{pfd}) \longrightarrow \mathcal{P}\left(\overline{\mathbb{R}}^{2} \times\{1,2,3,4\} \times \mathbb{N}\right) \\
\bigoplus_{\alpha \in A} I_{\alpha} \longmapsto \bigcup_{\alpha \in A}\left[\left\{\left(\inf I_{\alpha}, \sup I_{\alpha}\right)\right\} \times\left\{f\left(I_{\alpha}\right)\right\} \times\{m(i)\}\right]
\end{gathered}
$$

where $m(i)$ denotes the multiplicity of the direct summand $I_{\alpha}$. This map is an injection, hence (pfd) is a set.

Proposition 3.26. The class (eph) is not a set.

Proof For a cardinal $c$, let $F_{c}=\bigoplus_{\alpha \in c}[0,0]$. That is, $F_{c}$ is the k-vector space generated by $c$. For $c \neq d, F_{c} \approx F_{d}$. Thus we have an injection from the proper class of cardinals into (eph).

Corollary 3.27. Since (eph) is not a set, neither are (id) (qtame) and (pm).

3.6. Interval-decomposable persistence modules of arbitrary cardinality Motivated by the desire to have a set of persistence modules that contains all of the sets of persistence modules in Section 3.5 and the proofs of Proposition 3.22 and 3.25 , we make the following definition.

Definition 3.28. Given a cardinal $\kappa$, let $(\kappa$-id) denote the class of persistence modules isomorphic to $\bigoplus_{\alpha \in A} I_{\alpha}$ where $I_{\alpha}$ is an interval module and the cardinality of $A$ is at most $\kappa$. As a special case, and to avoid confusion with our previously defined notation, let (rid) denote the class of interval-decomposable persistence modules with at most the cardinality of $\mathbb{R}$-many summands.

By definition, (cid) $\subset$ (rid) and by Lemma 3.24, (pfd) $\subset$ (rid).

Proposition 3.29. For any cardinal $\kappa$, the class $(\kappa$-id) is a set.

Proof The proof is the same as the proof of Proposition 3.22 , replacing $\overline{\mathbb{N}}$ with $\kappa$.

\section{TOPOLOGICAL PROPERTIES}

Since we are interested in studying topological spaces of persistence modules, we will for the most part restrict ourselves to the sets in Figure 3. We will consider the basic topological properties of these sets with the topology induced by the interleaving metric. 


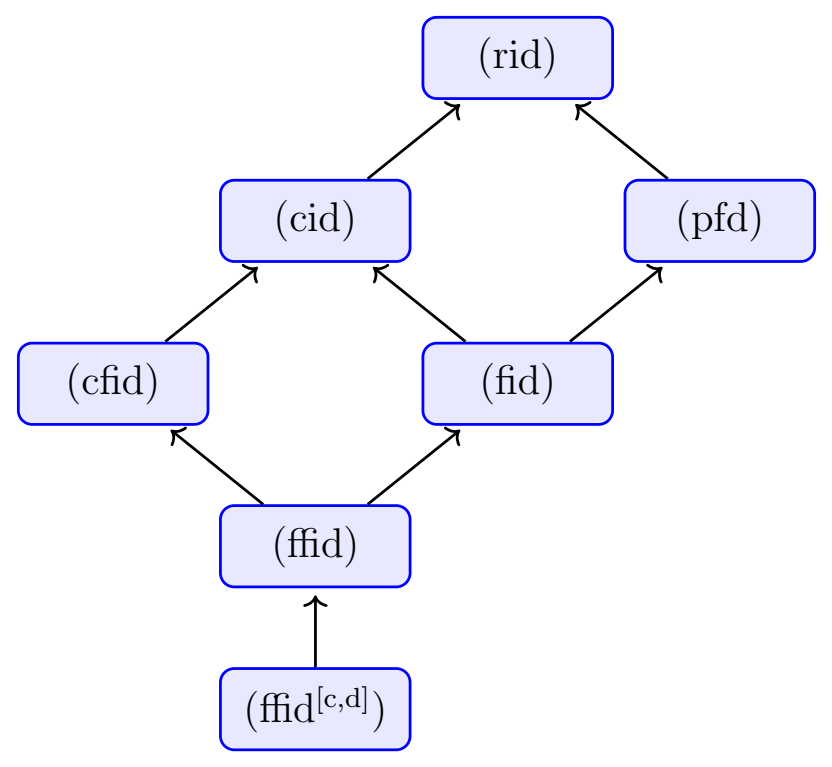

Figure 3. Sets of metric spaces, each with the topology induced by the interleaving metric.

4.1. Open subsets In this section we consider which of the inclusion maps in Figure 3 are inclusions of open subsets. Recall that in a pseudometric space $X$, a subset $A \subset X$ is said to be open if for all $a \in A$, there exists $\varepsilon>0$ such that $B_{\varepsilon}(a) \subset A$.

Proposition 4.1. Among the inclusion maps in Figure [3, only the inclusions (ffid) $\hookrightarrow$ (fid) and (cfid) $\hookrightarrow$ (cid) are inclusions of open subsets.

Proof Let $M \in$ (ffid) and $N \in$ (fid) \(ffid). Then $N$ is isomorphic to a direct sum of interval modules, at least one of which is unbounded. It follows that $d_{I}(M, N)=\infty$. Thus (ffid) is an open subset of (fid). The same argument shows that (cfid) is an open subset of (cid). For each of the following inclusions $\mathcal{A} \subset \mathcal{B}$ we show that for all $M \in \mathcal{A}$ and for all $\varepsilon>0$, there is an $N \in \mathcal{B} \backslash \mathcal{A}$ such that $d_{I}(M, N)<\varepsilon$. Therefore $\mathcal{A}$ is not an open subset of $\mathcal{B}$.

- (ffid $\left.{ }^{[\mathrm{c}, \mathrm{d}]}\right) \subset$ (ffid). Let $N=M \oplus[d, d+2 \varepsilon)$.

- (ffid) $\subset$ (cfid). Let $N=M \oplus \bigoplus_{k=1}^{\infty}[0,2 \varepsilon)$.

- (fid) $\subset$ (cid). Let $N=M \oplus \bigoplus_{k=1}^{\infty}[0,2 \varepsilon)$.

- (fid) $\subset$ (pfd). Let $N=M \oplus \bigoplus_{k=1}^{\infty}[k, k+2 \varepsilon)$.

- (cid) $\subset$ (rid). Let $N=M \oplus \bigoplus_{\mathbb{R}}[0,2 \varepsilon)$.

- $(\mathrm{pfd}) \subset($ rid $)$. Let $N=M \oplus \bigoplus_{k=1}^{\infty}[0,2 \varepsilon)$.

Remark 4.2. While (ffid ${ }^{[\mathrm{c}, \mathrm{d}]}$ ) is not an open subset of (ffid), if we restrict (ffid) to direct sums of interval modules whose intervals are contained in an open interval $(c, d)$, then we obtain an open subset of (ffid).

\subsection{Separation}

Proposition 4.3. Any set of ephemeral persistence modules with the interleaving distance has the indiscrete topology. 
Proof Let $S$ be a set of ephemeral persistence modules. By Lemma 3.6, each $M \in(\mathrm{eph})$ has $d_{I}(M, 0)=0$. So for $M, N \in S$, by the triangle inequality, $d_{I}(M, N)=0$. Thus for all $M \in S$ and for all $\varepsilon>0, B_{\varepsilon}(M) \supseteq S$.

Lemma 4.4. Let $M$ be a persistence module let $r \in \mathbb{R}$. Then $d_{I}(M, M \oplus[r, r])=0$.

A topological is said to be a $T_{0}$-space (or a Kolmogorov space), if for any pair of distinct elements in the space there exists at least one open set which contains one of them but not the other.

Proposition 4.5. Let $c<d$. Then $\left(\mathrm{ffid}^{[\mathrm{c}, \mathrm{d}]}\right)$ is not a $T_{0}$-space.

Proof Apply Lemma 4.4 to $M=[a, b)$ where $c \leq a<b \leq d$, and $r=\frac{c+d}{2}$. Then $M^{\prime}=$ $M \oplus[r, r] \in\left(\right.$ ffid $\left.^{[\mathrm{c}, \mathrm{d}]}\right)$ and there does not exist an open neighborhood $U$ of $M$ that does not contain $M^{\prime}$ and vice versa.

Since (ffid ${ }^{[c, d]}$ ) is a subspace of any the other spaces in Figure 3, we obtain the following.

Corollary 4.6. None of the spaces in Figure 3 are $T_{0}$.

4.3. Compactness Let $X$ be an extended pseudometric space. Then a subset $S \subset X$ is totally bounded if and only if for each $\varepsilon>0$, there exists a finite subset $F=\left\{x_{1}, x_{2}, \ldots, x_{n}\right\} \subset$ $X$ such that $S \subset \cup_{i=1}^{n} B_{\varepsilon}\left(x_{i}\right)$. Such a union is called a finite $\varepsilon$-cover.

Lemma 4.7. The space (ffid $\mathrm{d}^{[\mathrm{c}, \mathrm{d}]}$ ) is not totally bounded.

Proof Let $\varepsilon<\frac{d-c}{2}$. For $n \geq 0$ consider $M_{n}=\bigoplus_{k=1}^{n}[c, d)$. Then for $m \neq n, d_{I}\left(M_{m}, M_{n}\right)=$ $\frac{d-c}{2}$. Therefore (ffid $\left.{ }^{[\mathrm{c}, \mathrm{d}]}\right)$ does not have a finite $\varepsilon$-cover.

An open cover of a topological space $X$ is a collection of open sets $\mathcal{O}=\left\{O_{i}\right\}_{i \in I}$ of $X$ such that $\cup_{i \in I} O_{i}=X$. A topological spaces is compact if every open cover has a finite subcover. We say that a topological space is locally compact if each point has a compact neighborhood, where by a neighborhood of a point $p \in X$ we mean a subset $V \subset X$ such that there exists an open set $p \in U \subset V$.

Proposition 4.8. Any of element in (ffid $\left.{ }^{[\mathrm{c}, \mathrm{d}]}\right)$ does not have a compact neighborhood.

Proof Let $M \cong \bigoplus_{j=1}^{q} I_{j}$ with $I_{j} \subset[c, d]$. Suppose that $M$ has a compact neighborhood, $K$. Then there exists a real number $\varepsilon>0$ such that $M \in B_{\varepsilon}(M) \subset K$.

Choose $\delta>0$ such that $\delta<\varepsilon, \delta<d-c$ and $\delta<\frac{1}{4} \min _{j} \operatorname{diam} I_{j}$. Choose an interval $I$ of diameter $\delta$ contained in $[c, d]$. Consider for $n \in \mathbb{N}$, the persistence modules $M_{n}=$ $M \oplus \bigoplus_{k=1}^{n} I$. Then for each $n, d_{I}\left(M, M_{n}\right) \leq \frac{\delta}{2}$ so that the set $\left\{M_{n}\right\}_{n \in \mathbb{N}}$ is contained in $B_{\varepsilon}(M)$, and hence in $K$.

Let $M_{0}=M$. Then by the algebraic stability theorem [16], $d_{I}\left(M_{p}, M_{q}\right) \geq \frac{\delta}{2}$ for all $p>q \geq 0$. Now consider the open cover $\left\{B_{\frac{\delta}{6}}(N) \mid N \in K\right\}$ of $K$. It does not have a finite subcover, since there does not exist a persistence module $N$ such that $B_{\frac{\delta}{6}}(N)$ contains $M_{n}$ and $M_{m}$ for $m \neq n$.

Corollary 4.9. All of the spaces in Figure 3 are not locally compact.

An open covering $\mathcal{O}=\left\{O_{i}\right\}_{i \in I}$ of $X$ is locally finite if every $x \in X$ has a neighborhood which has a nonempty intersection with only finitely many of the open sets $\left\{O_{i}\right\}$. Given an 
open cover $\left\{O_{i}\right\}_{i \in I}$ of $X$, another open cover $\mathcal{V}=\left\{V_{j}\right\}_{j \in J}$ is called a refinement of $\mathcal{O}$ if for each $V$ in $\mathcal{V}$, there exists $O \in \mathcal{O}$ such that $V \subset O$. A topological space $X$ is said to be a paracompact if every open covering admits a locally finite refinement.

Lemma 4.10. An extended pseudometric space is paracompact.

Proof Let $(X, d)$ be an extended pseudometric space. Let $Y=X / \sim$ be the quotient space where the equivalence relation $\sim$ is defined on $X$ by $x \sim y \Leftrightarrow d(x, y)=0$. So $(Y, \rho)$ is an extended metric space where $\rho([x],[y])=d(x, y)$. Let $\pi: X \rightarrow Y$ denote the quotient map. Since $\pi$ maps the open ball $B_{r}(x)$ to the open ball $B_{r}([x])$ for all $x \in X$ and all $r>0$, it is an open map.

Now the equivalence relation on $Y$ given by $x \sim y \Leftrightarrow d(x, y)<\infty$ partitions $Y$ into a disjoint union of metric spaces, $Y=\coprod Y_{\alpha}$. Given an open cover $\mathcal{U}$ of $Y$, each open set in $\mathcal{U}$ is a disjoint union of open sets, each of which is in one of the $Y_{\alpha}$. This gives a refinement of $\mathcal{U}$ that is a disjoint union of open covers of each of the $Y_{\alpha}$. Each of these metric spaces is paracompact [36, Theorem 41.4]. Taking the disjoint union of the resulting locally finite refinements gives the desired locally finite refinement of $Y$.

Let $\mathcal{U}=\left\{U_{i}\right\}_{i \in I}$ be an open cover for $X$. Since $\pi$ is an open map $\left\{\pi\left(U_{i}\right)\right\}_{i \in I}$ forms an open cover for $Y$ and since $Y$ is paracompact there is a locally finite refinement $\mathcal{V}=\left\{V_{j}\right\}_{j \in J}$ for $\left\{\pi\left(U_{i}\right)\right\}_{i \in I}$. Then the open cover $\pi^{-1}(\mathcal{V})=\left\{\pi^{-1}\left(V_{j}\right)\right\}_{j \in J}$ is a locally finite refinement for $\mathcal{U}=\left\{U_{i}\right\}_{i \in I}$. Hence $X$ is paracompact.

\subsection{Path Connectedness}

Lemma 4.11. Let $S$ be an extended pseudometric space. Let $a, b \in S$ with $d(a, b)=\infty$. Then there does not exist a path in $S$ from a to $b$.

Proof Suppose there is a path $\gamma$ from $a$ to $b$ in $S$. Then $\gamma$ has a compact image. Therefore the cover $\left\{B_{1}(x) \mid x \in \gamma\right\}$ should have a finite subcover, which by the triangle inequality contradicts $d(a, b)=\infty$.

Corollary 4.12. The spaces of persistence modules (fid), (cid), (pfd), (rid), and (cfid) are not path connected.

Proof The first four of these sets contain both 0 and $[0, \infty)$ and $d_{I}(0,[0, \infty))=\infty$. The set (cfid) contains 0 and $\bigoplus_{k=1}^{\infty}[0, k)$ and $d_{I}\left(\bigoplus_{k=1}^{\infty}[0, k), 0\right)=\infty$.

Lemma 4.13. Let I be a finite interval. There exists a path in (ffid $\left.{ }^{[\mathrm{c}, \mathrm{d}]}\right)$ from $I$ to the zero module.

Proof Let $c=\inf I$ and $d=\sup I$. Let $M^{(0)}=I$ and $M^{(1)}=0$. For $0<t<1$, let $M^{(t)}=\left[c+t \frac{d-c}{2}, d-t \frac{d-c}{2}\right)$. Then for $0 \leq s \leq t \leq 1, d_{I}\left(M^{(s)}, M^{(t)}\right)=(t-s) \frac{d-c}{2}$. Thus $\gamma(t)=M^{(t)}$ is a (continuous) path from $I$ to 0 .

With a similar argument we will show the following.

Proposition 4.14. The path component of the zero module in (fid) is (ffid).

Proof By Lemma 4.11, the path component of 0 in (fid) is contained in (ffid). It remains to show that any $M \in$ (ffid) is path connected to 0 . 
Let $M \cong \bigoplus_{k=1}^{N} I_{k}$, where $I_{k}$ is a finite interval. For $1 \leq k \leq N$, let $c_{k}=\inf I_{k}$ and $d_{k}=$ $\sup I_{k}$. Let $M^{(0)}=M$ and $M^{(1)}=0$. For $0<t<1$, let $M^{(t)}=\bigoplus_{k=1}^{N}\left[c_{k}+t \frac{d_{k}-c_{k}}{2}, d-t \frac{d_{k}-c_{k}}{2}\right)$. Then for $0 \leq s \leq t \leq 1, d_{I}\left(M^{(s)}, M^{(t)}\right) \leq(t-s) \max _{1 \leq k \leq N} \frac{d_{k}-c_{k}}{2}$. So $M^{(t)}$ is a continuous path from $M$ to 0 .

Remark 4.15. It is not the case that the path component of the zero module in (cid) is (cfid), since (cfid) is not path connected. Since infinite intervals have infinite distance from the zero module, the path component of the zero module in (cid) is the same as the path component of the zero module in (cfid).

Proposition 4.16. The path component of 0 in (cfid), (pfd), and (rid) consists of modules $\bigoplus_{\alpha \in A} I_{\alpha}$, where $\sup _{\alpha \in A}$ length $\left(I_{\alpha}\right)<\infty$.

Proof Let $M=\bigoplus_{\alpha \in A} I_{\alpha}$. If $\sup _{\alpha \in A} \operatorname{length}\left(I_{\alpha}\right)=\infty$ then $d_{I}(0, M)=\infty$ and $M$ is not in the path component of 0 . If $\sup _{\alpha \in A} \operatorname{length}\left(I_{\alpha}\right)<\infty$ then the proof of Proposition 4.14 (replacing max with sup) shows that $M$ is in the path component of 0 .

The paths in the previous proposition may be used to show that the following spaces are nullhomotopic.

Proposition 4.17. The spaces (ffid ${ }^{[\mathrm{c}, \mathrm{d}]}$ ) and (ffid) and the path component of 0 of (cfid), (pfd) and (rid) are contractible to the zero module.

Proof Let $S$ denote either (ffid $\left.{ }^{[\mathrm{c}, \mathrm{d}]}\right)$, (ffid) or the path component of 0 in (cfid), (pfd), or (rid). Assume $M \cong \bigoplus_{k \in A} I_{k}$, where $A$ is countable. Let $c_{k}=\inf I_{k}, d_{k}=\sup I_{k}$ and let $h_{k}=\frac{d_{k}-c_{k}}{2}$. Let $M^{(0)}=M, M^{(1)}=0$ and for $0<t<1, M^{(t)}=\bigoplus_{k \in A}\left[c_{k}+t h_{k}, d-t h_{k}\right)$.

We will use these paths to construct a homotopy from the identity map on $S$ to the constant map to the zero module. Define $H: S \times[0,1] \rightarrow S$ by $(M, t) \mapsto M^{(t)}$. Let $H_{t}=H(-, t)$. Then $H_{0}=1_{S}$ and $H_{1}=0$. It remains to show that $H$ is continuous. Let $(M, t) \in S \times[0,1]$. Given $\varepsilon>0$, choose $\delta=\frac{\varepsilon}{1+d_{I}(M, 0)}$. Let $d$ denote the product metric on $S \times[0,1]$. Whenever $(N, s) \in S \times[0,1]$ satisfies $d((M, t),(N, s))<\delta, d_{I}(M, N)<\delta$ and $|t-s|<\delta$. Furthermore

$$
\begin{aligned}
d_{I}\left(M^{(t)}, N^{(s)}\right) \leq d_{I}\left(M^{(t)}, M^{(s)}\right)+d_{I}\left(M^{(s)}, N^{(s)}\right) & \\
& \leq|t-s| d_{I}(M, 0)+s d_{I}(M, N) \leq \delta d_{I}(M, 0)+\delta=\varepsilon
\end{aligned}
$$

which completes the proof.

4.5. Separability A topological space is said to be separable if it has a countable dense subset.

Theorem 4.18. The spaces (fid), (ffid) and (ffid $\mathrm{d}^{[\mathrm{c}, \mathrm{d}]}$ ) are separable.

Proof First we will show that (ffid) is separable. Let

$$
D_{n}=\left\{\bigoplus_{i=1}^{n}\left(p_{i}, q_{i}\right) \in(\text { fid }) \mid p_{i}, q_{i} \in \mathbb{Q}, p_{i}<q_{i}\right\}
$$

and then consider

$$
D=\bigcup_{\substack{i=1 \\ 18}}^{\infty} D_{n}
$$




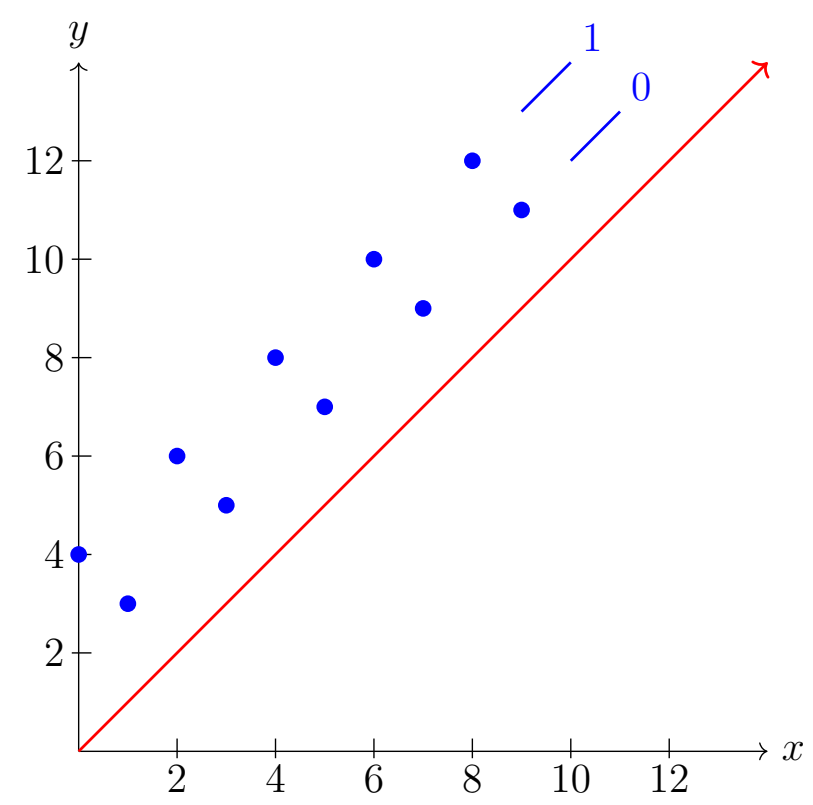

FiguRE 4. Persistence modules corresponding to binary sequences, which are used in the proof of Theorem 4.20.

Then $D$ is countable and $D$ is dense in (ffid) since every open ball of every persistence module in (ffid) contains an element of $D$.

This proof also works for (fid) if we allow the intervals in (4.19) to be infinite, and it works for (ffid ${ }^{[c, d]}$ ) if we restrict the intervals in (4.19) to be subintervals of $[c, d]$.

Theorem 4.20. The spaces (cfid), (cid), (pfd), and (rid) are not separable. The same is true for the subspace of (cid) with finite distance to 0 (which equals the subspace of (cfid) with finite distance to 0), and for (cid) $\cap$ (qtame) and (cfid) $\cap$ (qtame).

Proof We assign to each binary sequence, $\alpha=\left(\alpha_{n}\right)_{n \geq 1}$ where $\alpha_{n} \in\{0,1\}$, a persistence module. See Figure 4. For $n \geq 1$, define

$$
I_{n}^{(\alpha)}= \begin{cases}{[2 n-1,2 n+1),} & \alpha_{n}=0 \\ {[2(n-1), 2 n+2),} & \alpha_{n}=1\end{cases}
$$

and let $M_{\alpha}=\bigoplus_{n=1}^{\infty} I_{n}^{(\alpha)}$. Then $M_{\alpha}$ is a persistence module in (cfid), (cid), (pfd), (rid), and (qtame) and $d_{I}\left(M_{\alpha}, 0\right) \leq 2$.

The set $\left\{M_{\alpha} \mid \alpha\right.$ is a binary sequence $\}$ is uncountable and for all pairs of binary sequences $\alpha \neq \beta$, we have $d_{I}\left(M_{\alpha}, M_{\beta}\right)=1$. Then any dense subset of (cfid), (cid), (pfd), or (rid), contains a point in an open ball centered at each $M_{\alpha}$ of radius $\frac{1}{2}$ and thus cannot be countable. The same is true for the subspace of (cid) with finite distance to 0 , and for (cid) $\cap$ (qtame) and (cfid) $\cap$ (qtame).

4.6. Countability A topological space is said to be a first countable if it has a countable basis at each of its points.

Lemma 4.21. An extended pseudometric space is first countable. 
Proof Let $x$ be a point in the space. Then the countable collection of open balls $\left\{B_{\frac{1}{n}}(x) \mid n \in\right.$ $\mathbb{N}\}$ is the desired local base at $x$.

A space $X$ is compactly generated if a set $A \subset X$ is open if each $A \cap C$ is open in $C$ for each compact subspace $C \subset X$. Equivalently, a set $B \subset X$ is closed if each $B \cap C$ is closed in $C$ for each compact subspace $C \subset X$. The following is well known.

Lemma 4.22. If a space is first countable then it is compactly generated.

Proof For $B \subset X$, assume that $B \cap C$ is closed in $C$ for each compact subspace $C \subset X$. Let $x$ be a limit point of $B$. That is, every neighborhood of $x$ contains point of $B$ other than $x$. Since $X$ is first countable, there is a sequence of points $\left(x_{i}\right)$ converging to $x$. Now $\left(x_{i}\right) \cup\{x\}$ is compact, so by assumption $B \cap\left(\left(x_{i}\right) \cup\{x\}\right)$ is closed in $\left(x_{i}\right) \cup\{x\}$. Since $\left(x_{i}\right) \subset B$ it follows that $x \in B$. Therefore $B$ is closed.

A topological space is said to be second countable if it has a countable basis. A topological space $X$ is said to be Lindelöf if every open cover of $X$ admits a countable subcover.

Lemma 4.23. For an extended pseudometric space the following properties are equivalent:

(1) second countable;

(2) separable; and

(3) Lindelöf.

Proof Let $X$ be an extended pseudometric space.

$(1) \Rightarrow(2)$ : Assume that $X$ has a countable basis $\left\{B_{i}\right\}$. For each $i$, choose $x_{i} \in B_{i}$. Then for each $x \in X$ and $r>0$, there exists $i$ such that $B_{i} \subset B_{r}(x)$. So $\left\{x_{i}\right\}$ is a countable dense subset of $X$.

$(2) \Rightarrow(3)$ : Assume that $X$ has a countable dense subset $\left\{x_{i}\right\}$. Let $\mathcal{U}$ be an open cover of $X$. For each $i$, choose $U_{i} \in \mathcal{U}$ with $x_{i} \in U_{i}$. Since $U_{i}$ is open, $U_{i} \supset B_{r_{i}}\left(x_{i}\right)$ for some $r_{i}>0$. Since $\left\{x_{i}\right\}$ is dense, $\left\{U_{i}\right\}$ is a countable subcover.

$(3) \Rightarrow(1)$ : Assume that $X$ has the Lindelöf property. For each $n \geq 1$, let $\mathcal{U}_{n}$ be a countable subcover of the open cover $\left\{B_{\frac{1}{n}}(x) \mid x \in X\right\}$. Then $\mathcal{U}:=\cup_{n} \mathcal{U}_{n}$ is a countable basis for $X$.

4.7. Completeness An extended pseudometric space is said to be complete if every Cauchy sequence converges (see the end of Section 2.4).

Theorem 4.24. The spaces (pfd), (fid), (ffid) and (ffid $\left.{ }^{[\mathrm{c}, \mathrm{d}]}\right)$ are not complete.

Proof For $n \geq 0$, let $M_{n}=\bigoplus_{k=0}^{n}\left[-\frac{1}{2^{k}}, \frac{1}{2^{k}}\right)$. Then the sequence $\left(M_{n}\right) \subset($ ffid $) \subset($ fid $) \subset($ pfd $)$, and $\left(M_{n}\right) \rightarrow M=\bigoplus_{k=0}^{\infty}\left[-\frac{1}{2^{k}}, \frac{1}{2^{k}}\right)$, which is not in (pfd).

We claim that there is no $N \in(\mathrm{pfd})$ such that $d_{I}(M, N)=0$. Assume $N \in(\mathrm{pfd})$. Then $\operatorname{rank} N(0)=R<\infty$. Thus for all $\varepsilon>0, \operatorname{rank} N(-\varepsilon \leq \varepsilon) \leq R$. Now for all $\varepsilon>0, M$ and $N$ are $\varepsilon$-interleaved, and thus $\operatorname{rank} M(-2 \varepsilon \leq 2 \varepsilon) \leq \operatorname{rank} N(-\varepsilon \leq \varepsilon) \leq R$, which is a contradiction.

If we adjust $M_{n}$ to lie in $[c, d]$, then the same argument shows that (ffid $\left.{ }^{[\mathrm{c}, \mathrm{d}]}\right)$ is not complete.

Theorem 4.25. In the class of persistence modules and the class of q-tame persistence modules, every Cauchy sequence has a limit. Furthermore, the space (cid) $\cap$ (qtame) is complete, and so is (cfid) $\cap$ (qtame). 
Proof Let $\left(M_{n}^{\prime}\right)_{n \geq 1}$ be a Cauchy sequence of persistence modules. For each $k \geq 0$, choose a natural number $n_{k}$ so that $d_{I}\left(M_{m}^{\prime}, M_{n}^{\prime}\right)<\frac{1}{2^{k}}$ for all $m, n \geq n_{k}$. Let $M_{k}$ denote $M_{n_{k}}^{\prime}$. Thus $\left(M_{k}\right)$ is a subsequence of $\left(M_{n}^{\prime}\right)$ so that for all $k \geq 0, M_{k}$ and $M_{k+1}$ are $\frac{1}{2^{k}}$-interleaved. By the definition of interleaving, there exist natural transformations $\varphi_{k}: M_{k} \Rightarrow M_{k+1} T_{\frac{1}{2^{k}}}$ and $\psi_{k}: M_{k+1} \Rightarrow M_{k} T_{\frac{1}{2^{k}}}$ such that the triangles corresponding to (2.10) commute.

Now we define shifted versions of $\varphi$ and $\psi$. For $k \geq 0$, let $\alpha^{k}=\varphi_{k} T_{-\frac{1}{2^{k-1}}}: M_{k-1} T_{-\frac{1}{2^{k-1}}} \Rightarrow$ $M_{k} T_{-\frac{1}{2^{k}}}$, and $\beta^{k}=\psi_{k} T_{\frac{1}{2^{k}}}: M_{k} T_{\frac{1}{2^{k}}} \Rightarrow M_{k-1} T_{\frac{1}{2^{k-1}}}$. Let $a \in \mathbb{R}$. For every $k \geq 1, \alpha_{a}^{k}:$ $M_{k-1}\left(a-\frac{1}{2^{k-1}}\right) \rightarrow M_{k}\left(a-\frac{1}{2^{k}}\right)$ and $\beta_{a}^{k}: M_{k}\left(a+\frac{1}{2^{k}}\right) \rightarrow M_{k-1}\left(a+\frac{1}{2^{k-1}}\right)$. Thus we have a direct system of vector spaces

$$
M_{0}(a-1) \stackrel{\alpha_{a}^{1}}{\longrightarrow} M_{1}\left(a-\frac{1}{2}\right) \stackrel{\alpha_{a}^{2}}{\longrightarrow} M_{2}\left(a-\frac{1}{4}\right) \stackrel{\alpha_{a}^{3}}{\longrightarrow} M_{3}\left(a-\frac{1}{8}\right) \stackrel{\alpha_{a}^{4}}{\longrightarrow} \cdots
$$

and an inverse system of vector spaces

$$
\cdots \stackrel{\beta_{a}^{4}}{\longrightarrow} M_{3}\left(a+\frac{1}{8}\right) \stackrel{\beta_{a}^{3}}{\longrightarrow} M_{2}\left(a+\frac{1}{4}\right) \stackrel{\beta_{a}^{2}}{\rightarrow} M_{1}\left(a+\frac{1}{2}\right) \stackrel{\beta_{a}^{1}}{\longrightarrow} M_{0}(a+1)
$$

given in Figure 5. Note that it follows from the definition of interleaving that each of the trapezoids in Figure 5 commute. Let $A(a)$ be the colimit (i.e. direct limit) of (4.26),

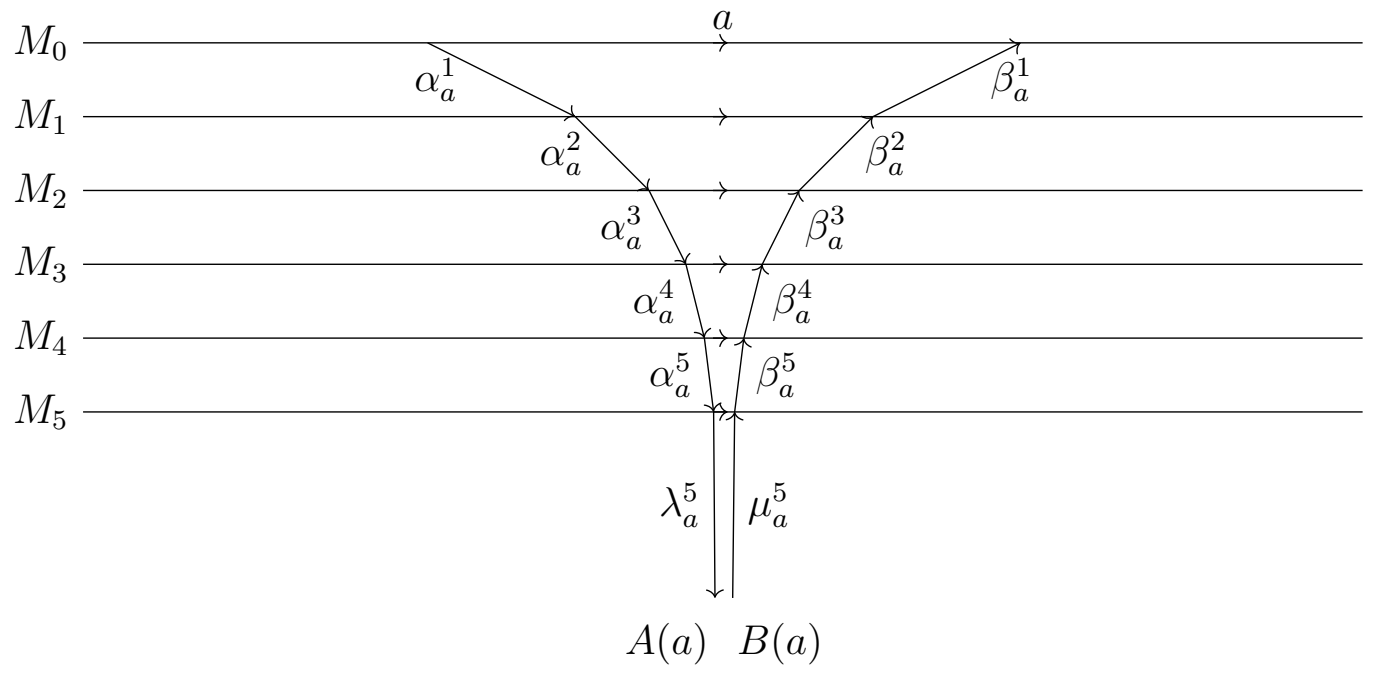

FiguRE 5. A direct system of vector spaces and an inverse system of vector spaces in a Cauchy sequence of persistence modules.

and let $B(a)$ be the limit (i.e. inverse limit) of (4.27). For each $k \geq 0$, we have maps $\lambda_{a}^{k}: M_{k}\left(a-\frac{1}{2^{k}}\right) \rightarrow A(a)$ and $\mu_{a}^{k}: B(a) \rightarrow M_{k}\left(a+\frac{1}{2^{k}}\right)$. By the universal properties of the colimit and the limit, we have a map $\theta_{a}: A(a) \rightarrow B(a)$, and

$$
\mu_{a}^{k} \theta_{a} \lambda_{a}^{k}=M_{k}\left(a-\frac{1}{2^{k}} \leq a+\frac{1}{2^{k}}\right) .
$$

Let $M(a)$ denote the image of $\theta_{a}$. Thus, $\theta_{a}$ factors as follows.

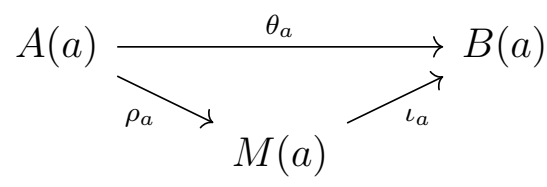


Now observe that all of these constructions are functorial. Thus, we have persistence modules $A, B$ and $M$. We also have natural transformations $\lambda^{k}: M_{k} T_{-\frac{1}{2^{k}}} \Rightarrow A$ and $\mu^{k}: B \Rightarrow$ $M_{k} T_{\frac{1}{2^{k}}}$. In addition we have the following commutative diagram of natural transformations.

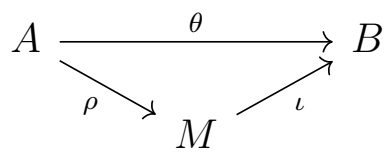

These fit into the commutative diagram in Figure 6, where we have corresponding arrows for all $a \in \mathbb{R}$.

$A, B, M, \operatorname{rad} M$

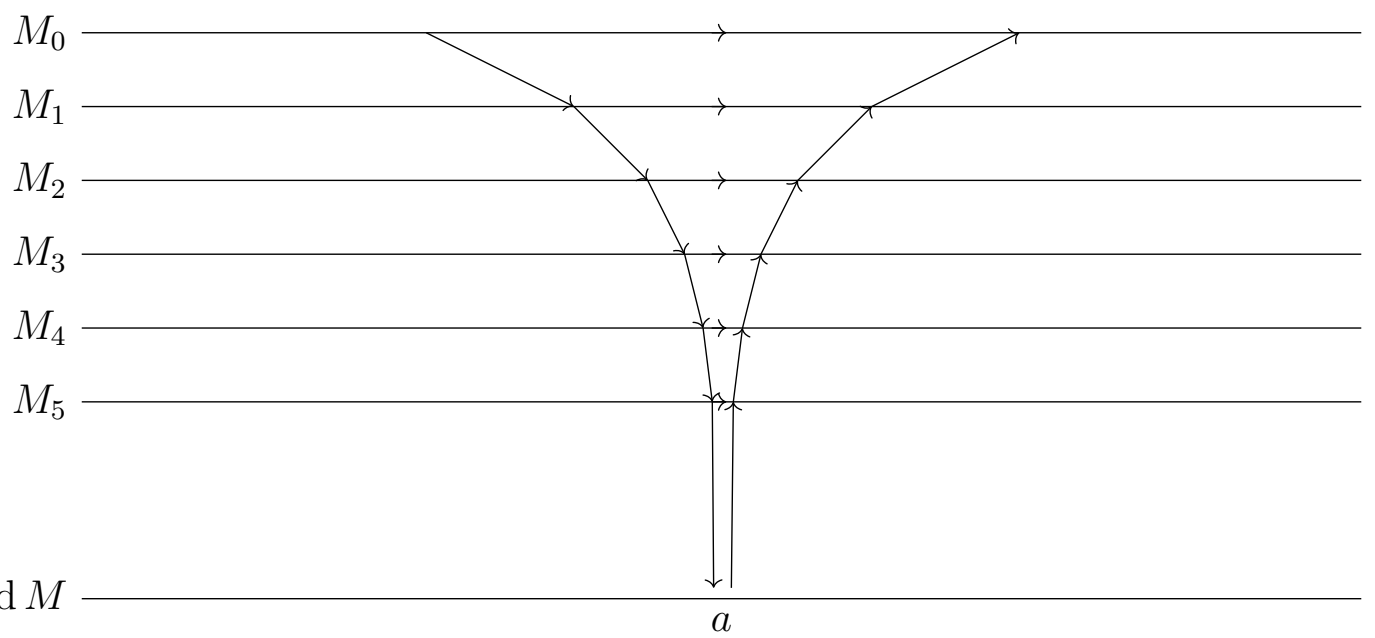

Figure 6. A particular subsequence of a Cauchy sequence of persistence modules and some persistence modules in the limit.

Let $a \in \mathbb{R}$ and $k \geq 1$. Define $b=a+\frac{1}{2^{k-1}}$. Then we have the following bi-infinite sequence.

$$
\begin{aligned}
\cdots \stackrel{\beta_{a}^{k+3}}{\longrightarrow} M_{k+2}\left(a+\frac{1}{2^{k+2}}\right) \stackrel{\beta_{a}^{k+2}}{\longrightarrow} & M_{k+1}\left(a+\frac{1}{2^{k+1}}\right) \stackrel{\beta_{a}^{k+1}}{\longrightarrow} M_{k}\left(a+\frac{1}{2^{k}}\right) \\
\stackrel{\alpha_{b}^{k+1}}{\longrightarrow} & M_{k+1}\left(b-\frac{1}{2^{k+1}}\right) \stackrel{\alpha_{b}^{k+2}}{\longrightarrow} M_{k+2}\left(b-\frac{1}{2^{k+2}}\right) \stackrel{\alpha_{b}^{k+3}}{\longrightarrow} \cdots
\end{aligned}
$$

Notice that the left part of this sequence is an initial part of (4.27) and the right part of this sequence is a terminal part of (4.26). It follows that (4.30) has limit $B(a)$ and colimit $A(b)$, and there is an induced map $\nu: B(a) \rightarrow A(b)$. We obtain the commutative diagram in Figure 7 .

By the universal properties of limit and colimit, we have the following commutative diagram.

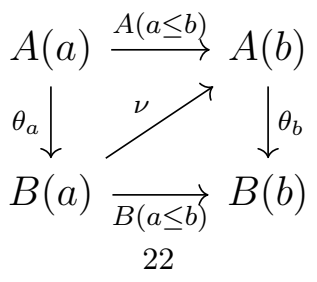




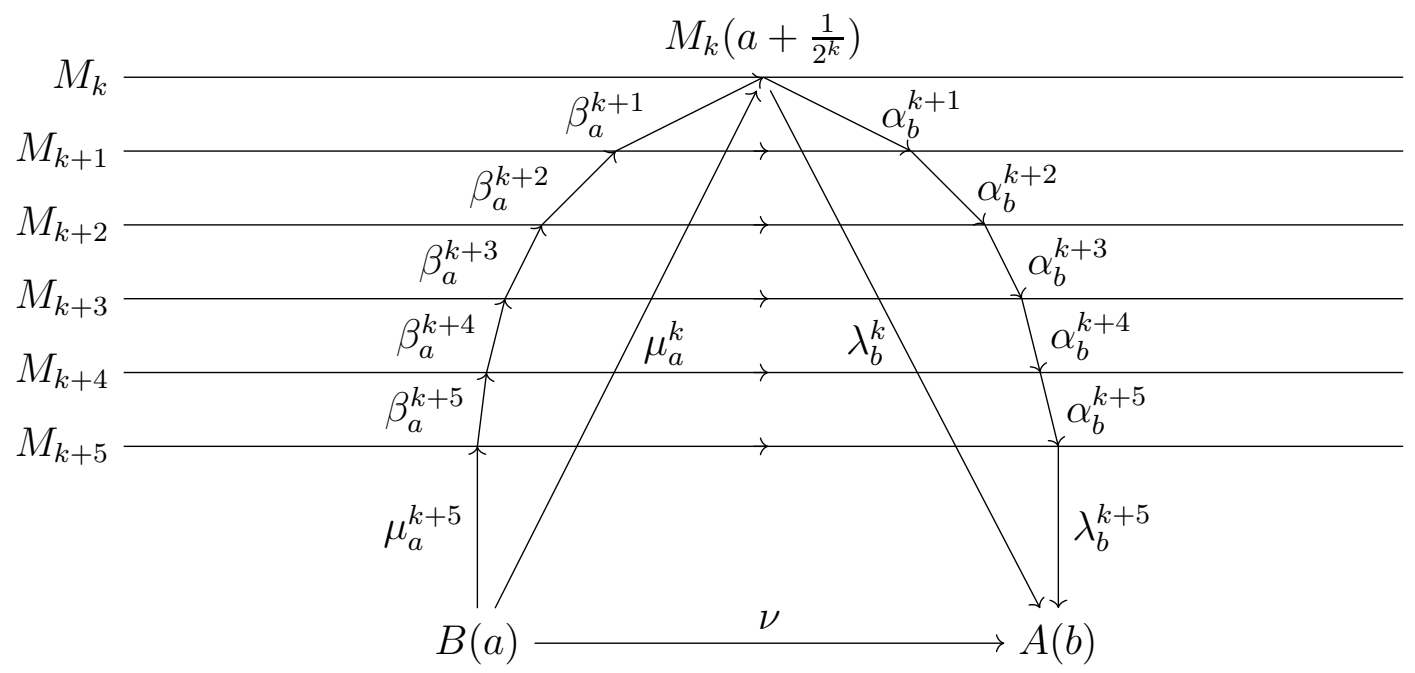

Figure 7. The bi-infinite sequence in (4.30), its limit and colimit, and three induced maps.

By the commutativity of the bottom right part of this diagram, we have that im $B(a \leq b) \subset$ $\operatorname{im} \theta_{b}$. So we have the following commutative diagram.

$$
M(a)=\operatorname{im} \theta_{a} \underset{M(a \leq b)}{\stackrel{\iota_{a}}{\longrightarrow} B(a) \underset{B(a \leq b)}{\longrightarrow} A(b)} \underset{\operatorname{im}_{b} \theta_{b}=M(b)}{\nu^{\rho_{b}}}
$$

Thus

$$
\rho_{b} \nu \iota_{a}=\left.B(a \leq b)\right|_{\operatorname{im} \theta_{a}}=M(a \leq b) .
$$

Now consider the following natural transformations.

$$
\begin{gathered}
\left(\rho \lambda^{k}\right) T_{\frac{1}{2^{k}}}: M_{k} \Rightarrow M T_{\frac{1}{2^{k}}} \\
\mu^{k} \iota: M \Rightarrow M_{k} T_{\frac{1}{2^{k}}}
\end{gathered}
$$

We claim that these natural transformations provide an interleaving (Section 2.3). That is,

$$
\left(\left(\mu^{k} \iota\right) T_{\frac{1}{2^{k}}}\right)\left(\left(\rho \lambda^{k}\right) T_{\frac{1}{2^{k}}}\right)=M_{k} \eta_{\frac{1}{2^{k-1}}}, \text { and }\left(\left(\rho \lambda^{k}\right) T_{\frac{1}{2^{k-1}}}\right)\left(\mu^{k} \iota\right)=M \eta_{\frac{1}{2^{k-1}}}
$$

where $\eta$ is the natural transformation defined in Section 2.3 .

A pair of natural transformations are equal if and only if their components are equal. We remark that for a natural transformations $\alpha$ and $\beta$, the natural transformation $\alpha T_{x}$ has components $\left(\alpha T_{x}\right)_{a}=\alpha_{a+x}$, and the natural transformation $\beta \alpha$ has components $(\beta \alpha)_{a}=$ $\beta_{a} \alpha_{a}$.

Let $a \in \mathbb{R}$. We will verify the identities in (4.34) using the $a$ component. For the left hand side of the first identity, we have

$$
M(a) \stackrel{\lambda_{a+\frac{1}{2^{k}}}}{\longrightarrow} A\left(a+\frac{1}{2^{k}}\right) \stackrel{\rho_{a+\frac{1}{2^{k}}}}{\longrightarrow} M\left(a+\frac{1}{2^{k}}\right) \stackrel{\iota_{a+\frac{1}{2^{k}}}^{\longrightarrow}}{\longrightarrow} B\left(a+\frac{1}{2^{k}}\right) \stackrel{\mu_{a+\frac{1}{2^{k}}}^{k}}{\longrightarrow} M\left(a+\frac{1}{2^{k-1}}\right)=M(b) .
$$


Using (4.29) the composition of the inner two maps equals $\theta_{a+\frac{1}{2^{k}}}$. Then using (4.28) we see that the entire composition equals $M_{k}\left(a \leq a+\frac{1}{2^{k-1}}\right)$, as desired.

For the left hand side of the second identity, we have

$$
M(a) \stackrel{\iota_{a}}{\longrightarrow} B(a) \stackrel{\mu_{a}^{k}}{\longrightarrow} M\left(a+\frac{1}{2^{k}}\right) \stackrel{\lambda_{a+\frac{1}{2^{k-1}}}^{k}}{\longrightarrow} A\left(a+\frac{1}{2^{k-1}}\right) \stackrel{\rho_{a+\frac{1}{2^{k-1}}}^{k}}{\longrightarrow} M\left(a+\frac{1}{2^{k-1}}\right)=M(b) .
$$

Using the commutativity of the induced maps in Figure 7 , the composition of the inner two maps equals $\nu$. Then using (4.31), we see that the entire composition equals $M(a \leq b)$, as desired.

Thus (4.32) and (4.33) is a $\frac{1}{2^{k}}$-interleaving. Therefore $M$ is a limit of the sequence $\left(M_{k}\right)$ and hence also a limit of the Cauchy sequence $\left(M_{n}^{\prime}\right)$. Thus any Cauchy sequence of persistence modules has a limit.

Now assume that each of the $M_{n}^{\prime}$ are in (qtame). We will show that $M \in$ (qtame). Let $a<b$, and choose $k \geq 0$ so that $\frac{1}{2^{k-1}}<b-a$. Then the following diagram commutes.

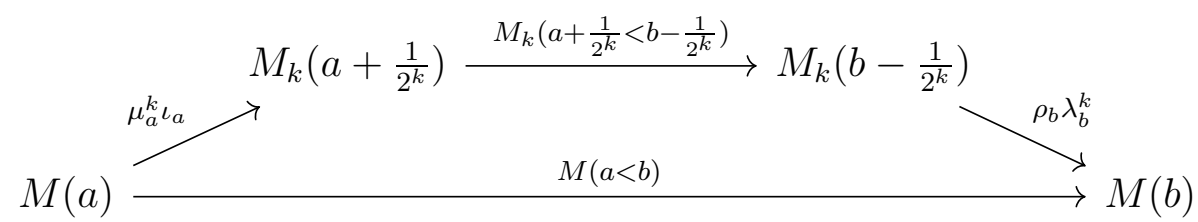

Since $M_{k}$ is q-tame, the top horizontal arrow has finite rank, and hence so does the bottom horizontal arrow. Thus $M \in$ (qtame). Therefore any Cauchy sequence of q-tame persistence modules has a limit.

Now since $M \in$ (qtame), by Theorem 3.8, $\operatorname{rad} M \in$ (cid) $\cap$ (qtame). By Proposition 3.7, $d_{I}(M, \operatorname{rad} M)=0$. Therefore by the triangle inequality, $\operatorname{rad} M$ is also a limit of the Cauchy sequence. Thus (cid) $\cap$ (qtame) is complete.

Finally, assume that in addition, each $M_{n}^{\prime} \in$ (cfid) $\cap$ (qtame). Since $M$ is $\frac{1}{2^{k}}$-interleaved with $M_{k}$, which does not contain any infinite intervals in its direct sum decomposition, neither does $M$. Therefore $\operatorname{rad} M$ also does not contain any infinite intervals in its direct sum decomposition. That is, $\operatorname{rad} M \in$ (cfid) $\cap$ (qtame).

Now we present a second, more concise proof of the main result in the previous proof.

Proof We may consider the diagram in Figure 5 to be a functor $M:(\mathbb{R} \times \mathbb{N}, \leq) \rightarrow \underline{\text { Vect }}_{\mathbf{k}}$, where $(\mathbb{R} \times \mathbb{N}, \leq)$ is the poset generated by the inequalities $(a, k) \leq(b, k)$, where $a \leq b \in \mathbb{R}$ and $k \geq 0$, and $(a, k-1) \leq\left(a+\frac{1}{2^{k}}, k\right)$ and $(a, k) \leq\left(a+\frac{1}{2^{k}}, k-1\right)$, where $a \in \mathbb{R}$ and $k \geq 1$.

Now extend this poset to $(\mathbb{R} \times \overline{\mathbb{N}}, \leq)$, by adding the generating inequalities $(a, \infty) \leq(b, \infty)$ for all $a \leq b$, and $\left(a-\frac{1}{2^{k}}, k\right) \leq(a, \infty)$ and $(a, \infty) \leq\left(a+\frac{1}{2^{k}}, k\right)$.

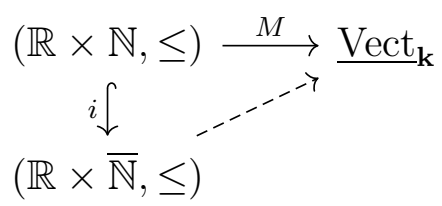

We can extend the functor $M$ to $(\mathbb{R} \times \overline{\mathbb{N}}, \leq)$ by taking either the left or the right Kan extension. We obtain functors corresponding to the diagram in Figure 6, where $A=\operatorname{Lan}_{i} M$ and $B=\operatorname{Ran}_{i} M$. Then there is a canonical map $\theta: A \Rightarrow B$, and the image of this map gives another extension of $M$. Abusing notation, let $M=\operatorname{im}(\theta):(\mathbb{R} \times \overline{\mathbb{N}}, \leq) \rightarrow$ Vect $_{\mathbf{k}}$. 
For $k \in \overline{\mathbb{N}}$, let $M_{k}=M(-, k)$. Then by construction, $M_{\infty}$ is $\frac{1}{2^{k}}$-interleaved with $M_{k}$. Thus, $M_{\infty}$ is a limit of the Cauchy sequence.

4.8. Baire spaces Let $X$ be a topological space. A subspace $A \subset X$ has empty interior in $X$ if $A$ does not contain an open set in $X$. The space $X$ is said to be a Baire space if for any countable collection of closed sets in $X$ with empty interior in $X$, their union also has empty interior in $X$.

Theorem 4.35 (Baire category theorem). A complete extended pseudometric space is a Baire space.

Proof Let $X$ be an extended pseudometric space. Let $\left\{A_{n}\right\}$ be a countable collection of closed sets in $X$ with empty interior in $X$. We want to show that $\bigcup A_{n}$ has empty interior in $X$. Let $U$ be an open set in $X$. We will show that $U \not \subset \cup A_{n}$. We need an $x \in U$ such that for all $n, x \notin A_{n}$. By assumption, there is a $x_{1} \in U$ with $x_{1} \notin A_{1}$. Since $U$ is open and $A_{1}$ is closed, there is an $r_{1} \leq 1$ such that $B_{r_{1}}\left(x_{1}\right) \subset U$ and $B_{r_{1}}\left(x_{1}\right) \cap A_{1}=\emptyset$. Let $s_{1}=\frac{r_{1}}{2}$. Then $\overline{B_{s_{1}}\left(x_{1}\right)} \subset U$ and $\overline{B_{s_{1}}\left(x_{1}\right)} \cap A_{1}=\emptyset$. Given $B_{s_{n}}\left(x_{n}\right)$ with $\overline{B_{s_{n}}\left(x_{n}\right)} \cap A_{n}=\emptyset$, then by assumption, there is a $x_{n+1} \in B_{s_{n}}(x)$ with $x_{n+1} \notin A_{n+1}$. Since $B_{s_{n}}(x)$ is open and $A_{n+1}$ is closed, there is an $r_{n+1} \leq \frac{1}{n+1}$ with $B_{r_{n+1}}\left(x_{n+1}\right) \subset B_{s_{n}}\left(y_{n}\right)$ and $B_{r_{n+1}}\left(x_{n+1}\right) \cap A_{n+1}=\emptyset$. Let $s_{n+1}=\frac{r_{n+1}}{2}$. Then $\overline{B_{s_{n+1}}\left(x_{n+1}\right)} \subset \overline{B_{s_{n}}\left(y_{n}\right)}$ and $\overline{B_{s_{n+1}}\left(x_{n+1}\right)} \cap A_{n+1}=\emptyset$. Since $\overline{B_{s_{1}}\left(x_{1}\right)} \supset$ $\overline{B_{s_{2}}\left(x_{2}\right)} \supset \overline{B_{s_{3}}\left(x_{3}\right)} \supset \cdots$ and $\left(s_{n}\right) \rightarrow 0,\left(x_{n}\right)$ is a Cauchy sequence in $X$. Since $X$ complete, there exists a $x \in X$ such that $\left(x_{n}\right) \rightarrow x$. Since $x_{n} \in \overline{B_{s_{1}}\left(x_{1}\right)}$ for all $n, x \in \overline{B_{s_{1}}\left(x_{1}\right)} \subset U$. Also, for all $n$, the sequence $x_{n}, x_{n+1}, x_{n+2}, \ldots$ in $\overline{B_{s_{n}}\left(x_{n}\right)}$ converges to $x$, so $x \in \overline{B_{s_{n}}\left(x_{n}\right)}$. Thus $x \notin A_{n}$ for all $n$.

Corollary 4.36. Hence (cid) $\cap$ (qtame) and (cfid) $\cap$ (qtame) are Baire spaces.

4.9. Topological dimension Let $X$ be a topological space. A collection of subsets of $X$ has order $m$ if there is a point in $X$ contained in $m$ of the subsets, but no point of $X$ is contained in $m+1$ of the subsets. The topological dimension of $X$ (also called the Lebesgue covering dimension) is the smallest number $m$ such that every open cover of $X$ has a refinement (see Section 4.3) with order $m+1$.

Theorem 4.37. Let $N \geq 1$. There exists an $\varepsilon>0$ such that there is an isometric embedding of the cube $[0, \varepsilon]^{N}$ with the $L^{\infty}$ distance into (ffid ${ }^{[\mathrm{c}, \mathrm{d}]}$ ).

Proof Assume $[c, d]=[0,1]$. The proof for the general case is similar. Choose $\varepsilon<\frac{1}{100 N}$. Let $x=\left(x_{1}, \ldots, x_{N}\right) \in[0, \varepsilon]^{N}$. We will define a map $x \mapsto M=M(x)=\bigoplus_{i=1}^{N} I_{i}$, where each interval $I_{i}=I_{i}\left(x_{i}\right)$ depends only on $x_{i}$. We will choose $I_{1}, \ldots, I_{N}$ to be far from each other and far from the zero module but so that $I_{i}\left(x_{i}\right)$ is close to $I_{i}\left(x_{i}^{\prime}\right)$ for any $x_{i}, x_{i}^{\prime} \in[0, \varepsilon]$.

For $1 \leq i \leq N$, let $I_{i}=\left[\frac{i}{N}, \frac{i}{N}+\frac{1}{10 N}+x_{i}\right)$. Then $d_{I}\left(I_{i}\left(x_{i}\right), I_{i}\left(x_{i}^{\prime}\right)\right)=\left|x_{i}-x_{i}^{\prime}\right| \leq \frac{1}{100 N}$. Also $d_{I}\left(I_{i}, 0\right) \geq \frac{1}{20 N}$. Since for $i \neq j, I_{i}$ and $I_{j}$ are disjoint, and so we also have that $d_{I}\left(I_{i}, I_{j}\right) \geq \frac{1}{20 N}$. Therefore $d_{I}\left(M(x), M\left(x^{\prime}\right)\right)=\left\|x-x^{\prime}\right\|_{\infty}$.

Corollary 4.38. The topological dimension of all of the topological spaces of persistence modules in Figure 3 is infinite.

Proof Let $X$ be one of the spaces in Figure 3. Then by the previous theorem, for all $N \geq 1$, $\operatorname{dim} X \geq \operatorname{dim}[0, \varepsilon]^{N}=N$. Thus $\operatorname{dim} X=\infty$. 


\section{Open questions}

We end with some unresolved questions.

- Are (cid) and (cfid) complete?

- Can the results presented here be extended to multiparameter persistence modules and generalized persistence modules?

Acknowledgments The authors would like to that the anonymous referees for their helpful suggestions. In particular, we would like to thank the referee who contributed the proof that the enveloping distance from pointwise-finite dimensional persistence modules to q-tame persistence modules is zero. We also thank Alex Elchesen for proofreading an earlier draft of the paper. The first author would like to acknowledge the support of the Army Research Office, Award W911NF1810307, and the Southeast Center for Mathematics and Biology, an NSF-Simons Research Center for Mathematics of Complex Biological Systems, under National Science Foundation Grant No. DMS-1764406 and Simons Foundation Grant No. 594594.

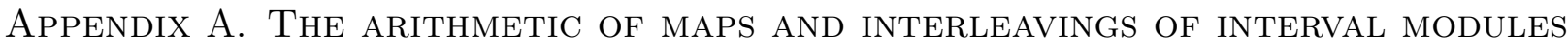

In this appendix, we give some basic results on interval modules, maps of interval modules, interleavings of interval modules, and neighborhoods of interval modules.

A.1. Some relations between intervals First we define some relations between intervals that will be useful in the following sections and describe some of their properties.

Recall that $I \subset \mathbb{R}$ is an interval if $a, c \in I$ and $a \leq b \leq c$ then $b \in I$. It follows that the intersection of two intervals is an interval.

Definition A.1. For $A, B \subset \mathbb{R}$, define the relation $A \leq B$ if

(1) for all $a \in A$ there is a $b \in B$ such that $a \leq b$, and

(2) for all $b \in B$ there is an $a \in A$ such that $a \leq b$.

Lemma A.2. This relation defines a partial order on intervals.

Proof Let $A, B$, and $C$ be intervals. $A \leq A$ since for all $a \in A, a \leq a$. Assume $A \leq B$ and $B \leq A$. Let $a \in A$. Then by Definition A.1 (1), there is $b \in B$ with $a \leq b$, and by Definition A.1 (2), there is $b^{\prime} \in B$ with $b^{\prime} \leq a$. Since $B$ is an interval $a \in B$. Thus $A \subset B$. Similarly $B \subset A$.

Finally assume $A \leq B$ and $B \leq C$. For all $a \in A$ there is a $b \in B$ with $a \leq b$ and $c \in C$ with $b \leq c$. Thus $a \leq c$. For all $c \in C$ there is a $b \in B$ with $b \leq c$ and $a \in A$ with $a \leq b$. Thus $a \leq c$. Therefore $A \leq C$.

Let us define another relation.

Definition A.3. For $A, B \subset \mathbb{R}$, define $A \prec B$ if for all $a \in A$ and $b \in B, a \leq b$.

Lemma A.4. Let $I$ and $J$ be disjoint, nonempty intervals. Then $J \leq I$ iff $J \prec I$.

Proof See Figure 8. Let $j \in J$. Then either condition implies that there is an $i \in I$ with $j \leq i$. The negation of either condition implies that there is an $i \in I$ with $i<j$. Since $I$ is an interval, this would imply that $j \in I$ which is a contradiction. 


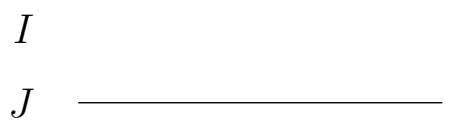

FiguRE 8. Two disjoint nonempty intervals.

Lemma A.5. If $J$ and $I$ are intervals with $J \leq I$ then $J \backslash(I \cap J)=J \backslash I$ is an interval and $I \backslash(I \cap J)=I \backslash J$ is an interval.

Proof Let $a, c \in J \backslash(I \cap J)$ and $a \leq b \leq c$. Since $J$ is an interval, $b \in J$. Since $c \in J$ there is a $d \in I$ with $c \leq d$. Since $c \notin I$ and $I$ is an interval, $b \notin I$. Thus $b \in J \backslash(I \cap J)$.

Let $a, c \in I \backslash(I \cap J)$ and $a \leq b \leq c$. Since $I$ is an interval $b \in I$. Since $a \in I$ there is a $x \in J$ with $x \leq a$. Since $a \notin J$ and $J$ is an interval, $b \notin J$. Thus $b \in I \backslash(I \cap J)$.

Lemma A.6. Let $I$ and $J$ be intervals with $J \leq I$. Then $J \backslash(I \cap J) \prec(I \cap J)$, and $(I \cap J) \prec I \backslash(I \cap J)$.

Proof First note that if either $A$ or $B$ is empty then $A \prec B$. Suppose $j \in J \backslash(I \cap J)$ and $i \in I \cap J$ with $i<j$. Since $J \leq I$, there is an $i^{\prime} \in I$ with $j \leq i^{\prime}$. Since $I$ is an interval, $j \in I$, which is a contradiction. Thus, for all $j \in J \backslash(I \cap J)$ and for all $i \in I \cap J, j \leq i$. That is, $J \backslash(I \cap J) \prec(I \cap J)$. Similarly, let $j \in I \cap J$ and $i \in I \backslash(I \cap J)$ with $i<j$. Again, since $J \leq I$, there is a $j^{\prime} \in J$ with $j^{\prime} \leq i$. Since $J$ is an interval, $i \in J$, which is a contradiction.

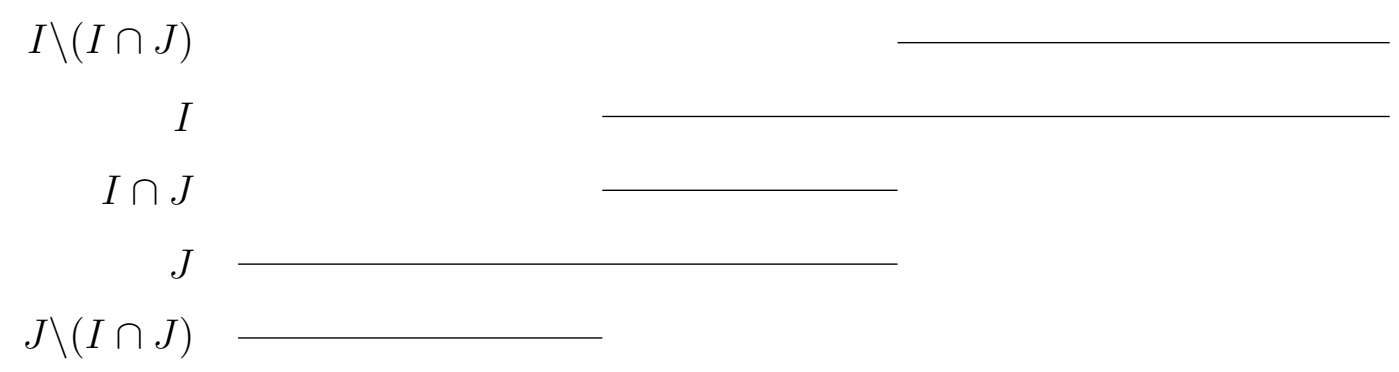

Figure 9. The interval modules in Lemma A.5, Lemma A.6, Proposition A.7, Lemma A.8, and Corollary A.9.

A.2. Nonzero maps of interval modules In this section we characterize nonzero maps of interval modules.

Proposition A.7. Let $I$ and $J$ be nonempty intervals. There is a nonzero map of persistence modules $f: I \rightarrow J$ if and only if $J \leq I$ and $I \cap J \neq \emptyset$.

Proof $(\Rightarrow)$ Assume $f \neq 0$. Then there is an $a \in \mathbb{R}$ such that $0 \neq f_{a}: I(a) \rightarrow J(a)$. Without loss of generality, assume that $f_{a}=1$. Thus $a \in I$ and $a \in J$. We need to check the conditions in Definition A.1. 
(11) For all $i \in I$ with $a \leq i$, the condition is satisfied by $a \in J$. For all $i \in I$ with $i \leq a$, we have the following commutative diagram,

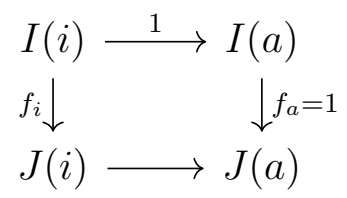

which implies that $i \in J$, and thus $J(i \leq a)=1$, and therefore $f_{i}=1$. (2) For all $j \in J$ with $j \leq a$, the condition is satisfied by $a \in I$. For all $j \in J$ with $a \leq j$, we have the following commutative diagram,

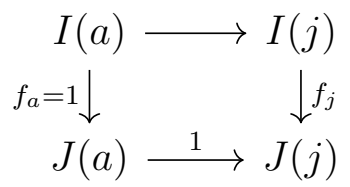

which implies that $j \in I, I(a \leq j)=1$, and $f_{j}=1$.

$(\Leftarrow)$ Define $f: I \rightarrow J$ by $f_{a}=1$ if $a \in I \cap J$, and $f_{a}=0$ otherwise. We claim that $f$ is a natural transformation. For $a \leq b$, we need to check that the diagram

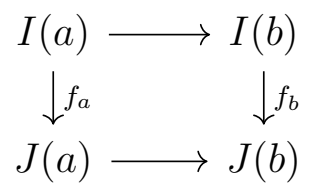

commutes. There are four cases to check. If $a, b \in I \cap J$, then all four maps are the identity and thus the diagram commutes. If $a, b \notin I \cap J$ then both vertical maps are zero and thus the diagram commutes.

If $a \in I \cap J$ and $b \notin I \cap J$ then by definition the left map is the identity and the right map is zero. If $b \in J$ then $b \notin I$, which implies, since $I$ is an interval, that for all $c \geq b$, $c \notin I$. But this contradicts Definition A.1 (1). Therefore $b \notin J$. Thus $J(b)=0$ and hence the diagram commutes.

If $a \notin I \cap J$ and $b \in I \cap J$, then $f_{a}=0$ and without loss of generality $f_{b}=1$. Again $a \in I$ implies $a \notin J$, which implies that for all $c \leq a, c \notin J$, which is a contradiction. Therefore $a \notin I$ which implies that $I(a)=0$ and thus the diagram commutes.

Lemma A.8. Assume there is a nonzero map $f: I \rightarrow J$ of interval modules. Then (up to isomorphism) $f_{a}=1$ if $a \in I \cap J$ and $f_{a}=0$ otherwise.

Proof Assume $f \neq 0$. The there is a $b \in I \cap J$ such that $f_{b}$ is nonzero. Without loss of generality, we may assume that $f_{b}=1$. Let $a \leq b \leq c \in I \cap J$. We have the following commutative diagram,

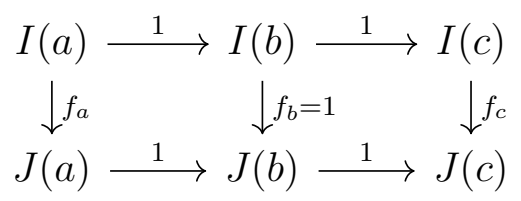

which implies that $f_{a}=1$ and $f_{c}=1$. Thus $f_{a}=1$ for all $a \in I \cap J$.

If $a \notin I \cap J$ then either $I(a)=0$ or $J(a)=0$, which implies that $f_{a}=0$. 
Corollary A.9. Let $f: I \rightarrow J$ be a nonzero map of interval modules. Then the image of $f$ is $I \cap J$, the kernel of $f$ is $I \backslash(I \cap J)$, the cokernel of $f$ is $J \backslash(I \cap J)$, and $f$ factors as follows.

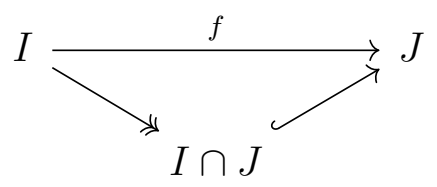

A.3. Interleavings of interval modules In this section we characterize interleavings of interval modules.

Definition A.10. Let $I$ be an interval and $\varepsilon \in \mathbb{R}$. Define the shifted interval $I[\varepsilon]$ by $x \in I[\varepsilon]$ if and only if $x+\varepsilon \in I$. For example, $[a, b)[\varepsilon]=[a-\varepsilon, b-\varepsilon)$.

The next lemma follows immediately from the definitions.

Lemma A.11. If $I$ is a nonempty interval and $\varepsilon \geq 0$, then $I[\varepsilon] \leq I$.

Definition A.12. Let $M$ be a persistence module and let $\varepsilon \in \mathbb{R}$. We define the shifted persistence module $M[\varepsilon]$ by $M[\varepsilon](a)=M(a+\varepsilon)$ and $M[\varepsilon](a \leq b)=M(a+\varepsilon \leq b+\varepsilon)$. That is, $M[\varepsilon]=M T_{\varepsilon}$.

We remark that these two definitions are compatible. If $I$ is an interval module and $\varepsilon \in \mathbb{R}$, then the shifted persistence module $I[\varepsilon]$ is the interval module on the interval $I[\varepsilon]$. Also note that $0[\varepsilon]=0$.

Let $I$ be an interval and $\varepsilon \geq 0$. If $I \cap I[\varepsilon] \neq \emptyset$, we denote the corresponding nonzero map from Proposition A.7 by $I^{(\varepsilon)}: I \rightarrow I[\varepsilon]$. If $I$ and $I[\varepsilon]$ are disjoint, we denote the zero map by $I^{(\varepsilon)}: I \rightarrow I[\varepsilon]$. In either case, $I^{(\varepsilon)}=I \eta_{\varepsilon}$.

Definition A.13. Given a map of persistence modules $\alpha: M \rightarrow N$ and $\varepsilon \in \mathbb{R}$, define $\alpha[\varepsilon]: M[\varepsilon] \rightarrow N[\varepsilon]$ by $\alpha[\varepsilon]_{a}=\alpha_{a+\varepsilon}$. That is, $\alpha[\varepsilon]=\alpha T_{\varepsilon}$.

As a special case of Definition 2.8, we have the following.

Definition A.14. Let $I$ and $J$ be interval modules and $\varepsilon \geq 0$. Then $I$ and $J$ are $\varepsilon$-interleaved if there exist maps $\varphi: I \rightarrow J[\varepsilon]$ and $\psi: J \rightarrow I[\varepsilon]$ such that $\psi[\varepsilon] \varphi=I^{(2 \varepsilon)}$ and $\varphi[\varepsilon] \psi=J^{(2 \varepsilon)}$.

Lemma A.15. If intervals satisfy $K \leq J \leq I$ then $I \cap K \subset J$.

Proof Let $x \in I \cap K$. Then there is a $j \in J$ such that $x \leq j$. Also, there is a $j^{\prime} \in J$ with $j^{\prime} \leq x$. Since $J$ is an interval, $x \in J$.

Lemma A.16. If $K \leq J \leq I$ then $I \cap K=(I \cap J) \cap(J \cap K)$.

Proof One direction is easy: $(I \cap J) \cap(J \cap K)=I \cap J \cap K \subset I \cap K$. The other direction follows from Lemma A.15.

Proposition A.17. Let $I$ and $J$ be interval modules and $\varepsilon \geq 0$. If $J[\varepsilon] \leq I$ and $I[\varepsilon] \leq J$ then $I$ and $J$ are $\varepsilon$-interleaved.

Proof Define $\varphi: I \rightarrow J[\varepsilon]$ by $\varphi_{x}=1$ if $x \in I \cap J[\varepsilon]$ and $\varphi_{x}=0$ otherwise. Similarly define $\psi: J \rightarrow I[\varepsilon]$ by $\psi_{x}=1$ if $x \in J \cap I[\varepsilon]$ and $\psi_{x}=0$ otherwise. We claim that these provide the desired $\varepsilon$-interleaving. 
First, $I^{(2 \varepsilon)}: I \rightarrow I[2 \varepsilon]$ is given by $I_{x}^{(2 \varepsilon)}=1$ if $x \in I \cap I[2 \varepsilon]$ and $I_{x}^{(2 \varepsilon)}=0$ otherwise. Next, $\psi[\varepsilon] \varphi_{x}=1$ if $x \in(I \cap J[\varepsilon]) \cap(J[\varepsilon] \cap I[2 \varepsilon])$ and $\psi[\varepsilon] \varphi_{x}=0$ otherwise. By Lemma A.16, these maps are equal. Similarly, $\varphi[\varepsilon] \psi=J^{(2 \varepsilon)}$.

Next, we define the erosion of a persistence module. Compare with [37, 39].

Definition A.18. Let $I$ be an interval or an interval module and $\varepsilon \geq 0$. We define the $\varepsilon$-erosion of $I$ to be $I^{-\varepsilon}=I[\varepsilon] \cap I[-\varepsilon]$.

Note that $I[\varepsilon] \leq I^{-\varepsilon} \leq I[-\varepsilon]$. See Figure 10 .

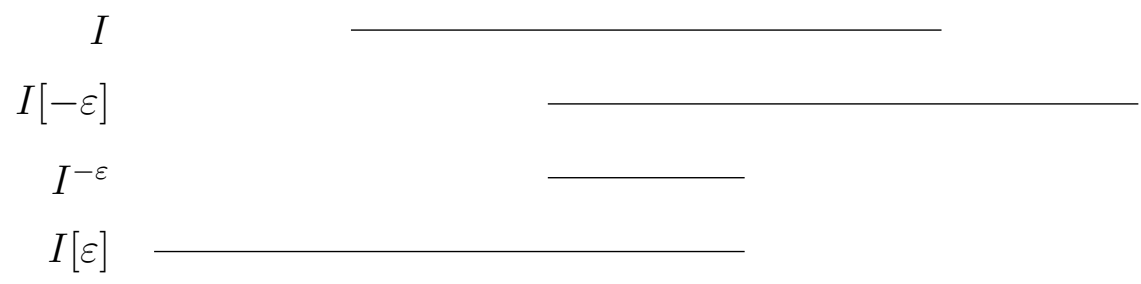

Figure 10. An interval module and its erosion.

Corollary A.19. If $J[\varepsilon] \leq I$ and $I[\varepsilon] \leq J$ then $I^{-\varepsilon} \subset J$ and $J^{-\varepsilon} \subset I$.

Proof It follows from the assumptions that we also have $J \leq I[-\varepsilon]$ and $I \leq J[-\varepsilon]$. So we have $J[\varepsilon] \leq I \leq J[-\varepsilon]$ and $I[\varepsilon] \leq J \leq I[-\varepsilon]$. The result follows from Lemma A.15.

Theorem A.20. Let $I$ and $J$ be interval modules and $\varepsilon \geq 0$. Then $I$ and $J$ are $\varepsilon$-interleaved if only if $I^{-\varepsilon} \subset J$ and $J^{-\varepsilon} \subset I$.

Proof $(\Rightarrow)$ Let $\varphi$ and $\psi$ be an $\varepsilon$-interleaving. If either $\varphi$ or $\psi$ are zero, then from Definition A.14, $I^{(2 \varepsilon)}$ and $J^{(2 \varepsilon)}$ are zero. It follows that $I^{-\varepsilon}$ and $J^{-\varepsilon}$ are both empty and the condition is satisfied. If both $\varphi$ and $\psi$ are nonzero, then by Proposition A.7, $J[\varepsilon] \leq I$ and $I[\varepsilon] \leq J$. The result follows from Corollary A.19.

$(\Leftarrow)$ We need to check four cases. (1) $I^{-\varepsilon}$ and $J^{-\varepsilon}$ are both empty. Then $I$ and $J$ are $\varepsilon$-interleaved by $\varphi=0$ and $\psi=0$.

(2) $I^{-\varepsilon}$ and $J^{-\varepsilon}$ are both nonempty. Let $a \in I[\varepsilon]$. Then there is an element $b \in I^{-\varepsilon} \subset J$ with $a \leq b$. Let $b \in I$. Then there is an element $a \in I^{-\varepsilon}[\varepsilon] \subset J[\varepsilon]$ with $a \leq b$. Let $a \in J[\varepsilon]$. Then there is an element $b \in J^{-\varepsilon} \subset I$ with $a \leq b$. Let $b \in J$. Then there is an element $a \in J^{-\varepsilon}[\varepsilon] \subset I[\varepsilon]$ with $a \leq b$. The result follows from Proposition A.17.

(3) $I^{-\varepsilon}$ is nonempty and $J^{-\varepsilon}$ is empty. Let $a \in I[\varepsilon]$. Then there is $b \in I^{-\varepsilon} \subset J$ with $a \leq b$. Since $J$ is shorter than $I$, it follows that $I[\varepsilon] \leq J$. Let $b \in I$. Then there is $a \in I^{-\varepsilon}[\varepsilon] \subset J[\varepsilon]$ with $a \leq b$. Since $J$ is shorter than $I$, it follows that $J[\varepsilon] \leq I$. The result follows from Proposition A.17.

(4) is the same as the third case.

A.4. Neighborhoods of interval modules Using Theorem A.20, one obtains a complete characterization of the interval modules within distance $\varepsilon$ of an interval module. 
Example A.21. Consider the interval module $[a, b)$ and let $\varepsilon \in\left[0, \frac{b-a}{2}\right)$. Then an interval module $I$ is $\varepsilon$-interleaved with $[a, b)$ if and only if $[a+\varepsilon, b-\varepsilon) \subset I \subset[a-\varepsilon, b+\varepsilon)$. Furthermore $B_{\varepsilon}([a, b))$ consists of those interval modules $I$ satisfying

$$
a-\varepsilon<\inf I<a+\varepsilon \text { and } b-\varepsilon<\sup I<b+\varepsilon .
$$

Example A.22. Consider the interval module $[a, b)$ and let $\varepsilon \geq \frac{b-a}{2}$. Then an interval $I$ is $\varepsilon$-interleaved with $[a, b)$ if and only if either $I \subset[a-\varepsilon, b+\varepsilon)$ or if for no $x \in \mathbb{R}$ do we have $[x-\varepsilon, x+\varepsilon] \subset I$. Furthermore, $B_{\varepsilon}([a, b))$ consists of those interval modules $I$ with either $a-\varepsilon<\inf I$ and $\sup I<b+\varepsilon$ or $\operatorname{diam} I<\varepsilon$.

Example A.23. Consider the interval module $[a, \infty)$ and let $\varepsilon \geq 0$. Then an interval module $I$ is $\varepsilon$-interleaved with $[a, \infty)$ if and only if $[a+\varepsilon, \infty) \subset I \subset[a-\varepsilon, \infty)$. Furthermore $B_{\varepsilon}([a, \infty))$ consists of interval modules $I$ satisfying $a-\varepsilon<\inf I<a+\varepsilon$.

\section{REFERENCES}

[1] Ulrich Bauer and Michael Lesnick. Induced matchings and the algebraic stability of persistence barcodes. J. Comput. Geom., 6(2):162-191, 2015.

[2] Ulrich Bauer and Michael Lesnick. Persistence diagrams as diagrams: A categorification of the stability theorem, 10 2016, 1610.10085.

[3] Håvard Bakke Bjerkevik and Magnus Bakke Botnan. Computational complexity of the interleaving distance. 12 2017, 1712.04281.

[4] Andrew J. Blumberg, Itamar Gal, Michael A. Mandell, and Matthew Pancia. Robust statistics, hypothesis testing, and confidence intervals for persistent homology on metric measure spaces. Found. Comput. Math., 14(4):745-789, 2014.

[5] Andrew J. Blumberg and Michael Lesnick. Universality of the homotopy interleaving distance. 05 2017, 1705.01690.

[6] Magnus Bakke Botnan and Michael Lesnick. Algebraic stability of zigzag persistence modules. 04 2016, 1604.00655 .

[7] Peter Bubenik. Statistical topological data analysis using persistence landscapes. Journal of Machine Learning Research, 16:77-102, 2015.

[8] Peter Bubenik, Vin de Silva, and Vidit Nanda. Higher interpolation and extension for persistence modules. SIAM Journal on Applied Algebra and Geometry, 1(1):272-284, 2017.

[9] Peter Bubenik, Vin de Silva, and Jonathan Scott. Metrics for Generalized Persistence Modules. Found. Comput. Math., 15(6):1501-1531, 2015.

[10] Peter Bubenik, Vin de Silva, and Jonathan Scott. Interleaving and gromov-hausdorff distance. 07 2017, 1707.06288.

[11] Peter Bubenik and Pawel Dlotko. A persistence landscapes toolbox for topological statistics. Journal of Symbolic Computation, 78:91 - 114, 2017.

[12] Peter Bubenik and Jonathan A. Scott. Categorification of persistent homology. Discrete Comput. Geom., 51(3):600-627, 2014.

[13] Gunnar Carlsson. Topology and data. Bull. Amer. Math. Soc. (N.S.), 46(2):255-308, 2009.

[14] Gunnar Carlsson and Afra Zomorodian. The theory of multidimensional persistence. Discrete Comput. Geom., 42(1):71-93, 2009.

[15] Frédéric Chazal, David Cohen-Steiner, Marc Glisse, Leonidas J. Guibas, and Steve Y. Oudot. Proximity of persistence modules and their diagrams. In Proceedings of the 25th annual symposium on Computational geometry, SCG '09, pages 237-246, New York, NY, USA, 2009. ACM.

[16] Frédéric Chazal, David Cohen-Steiner, Marc Glisse, Leonidas J. Guibas, and Steve Y. Oudot. Proximity of persistence modules and their diagrams. In Proceedings of the 25th annual symposium on Computational geometry, SCG '09, pages 237-246, New York, NY, USA, 2009. ACM.

[17] Frédéric Chazal, William Crawley-Boevey, and Vin de Silva. The observable structure of persistence modules. Homology Homotopy Appl., 18(2):247-265, 2016. 
[18] Frédéric Chazal, Vin de Silva, Marc Glisse, and Steve Oudot. The structure and stability of persistence modules. SpringerBriefs in Mathematics. Springer, [Cham], 2016.

[19] Frédéric Chazal, Vin de Silva, and Steve Oudot. Persistence stability for geometric complexes. Geom. Dedicata, 173:193-214, 2014.

[20] Frédéric Chazal and Bertrand Michel. An introduction to topological data analysis: fundamental and practical aspects for data scientists. 10 2017, 1710.04019.

[21] David Cohen-Steiner, Herbert Edelsbrunner, and John Harer. Stability of persistence diagrams. Discrete Comput. Geom., 37(1):103-120, 2007.

[22] Anne Collins, Afra Zomorodian, Gunnar Carlsson, and Leonidas J. Guibas. A barcode shape descriptor for curve point cloud data. Computers $\& 3$ Graphics, 28(6):881 - 894, 2004.

[23] William Crawley-Boevey. Decomposition of pointwise finite-dimensional persistence modules. J. Algebra Appl., 14(5):1550066, 8, 2015.

[24] Joshua Cruz. Cauchy sequences in categories with an interleaving distance. in preparation, 2018.

[25] Justin Curry. Sheaves, Cosheaves and Applications. PhD thesis, University of Pennsylvania, 2014.

[26] Vin de Silva, Elizabeth Munch, and Amit Patel. Categorified Reeb graphs. Discrete Comput. Geom., $55(4): 854-906,2016$.

[27] Vin de Silva, Elizabeth Munch, and Anastasios Stefanou. Theory of interleavings on $[0, \infty)$-actegories. 062017.

[28] Peter Gabriel. Unzerlegbare Darstellungen. I. Manuscripta Math., 6:71-103; correction, ibid. 6 (1972), 309, 1972.

[29] Robert Ghrist. Homological algebra and data. to appear, IAS/Park City Volume.

[30] Robert Ghrist. Barcodes: the persistent topology of data. Bull. Amer. Math. Soc. (N.S.), 45(1):61-75, 2008.

[31] Michael Lesnick. The theory of the interleaving distance on multidimensional persistence modules. Found. Comput. Math., 15(3):613-650, 2015.

[32] Killian Meehan and David Meyer. Interleaving distance as a limit. 10 2017, 1710.11489.

[33] Killian Meehan and David Meyer. An isometry theorem for generalized persistence modules. 10 2017, 1710.02858 .

[34] Yuriy Mileyko, Sayan Mukherjee, and John Harer. Probability measures on the space of persistence diagrams. Inverse Problems, 27(12):124007, 22, 2011.

[35] Elizabeth Munch and Bei Wang. Convergence between categorical representations of reeb space and mapper. 12 2015, 1512.04108.

[36] James R. Munkres. Topology: a first course. Prentice-Hall, Inc., Englewood Cliffs, N.J., 1975.

[37] Amit Patel. Generalized persistence diagrams. Journal of Applied and Computational Topology, 1(3):397-419, Jun 2018

[38] Jose A. Perea, Elizabeth Munch, and Firas Khasawneh. Approximating continuous functions on the space of persistence diagrams. in preparation, 2018.

[39] Ville Puuska. Erosion distance for generalized persistence modules. 10 2017, 1710.01577.

[40] Katharine Turner, Yuriy Mileyko, Sayan Mukherjee, and John Harer. Fréchet means for distributions of persistence diagrams. Discrete Comput. Geom., 52(1):44-70, 2014.

[41] Larry Wasserman. Topological data analysis. 09 2016, 1609.08227. arXiv:1609.08227 [stat.ME].

[42] Cary Webb. Decomposition of graded modules. Proc. Amer. Math. Soc., 94(4):565-571, 1985.

Department of Mathematics, University of Florida

E-mail address: peter.bubenik@ufl.edu

Department of Mathematics, Ege University

E-mail address: tane.vergili@ege.edu.tr 